\title{
3D simulations identifying the effects of varying the twist and field strength of an emerging flux tube
}

\author{
M. J. Murray ${ }^{1}$, A. W. Hood ${ }^{1}$, F. Moreno-Insertis ${ }^{2,3}$, K. Galsgaard ${ }^{4}$, and V. Archontis ${ }^{1}$ \\ ${ }^{1}$ School of Mathematics and Statistics, University of St. Andrews, North Haugh, St. Andrews, Fife, KY16 9SS, UK \\ e-mail: michelle@mcs.st-and.ac.uk \\ 2 Institúto de Astrofísica de Canarias, 38200 La Laguna (Tenerife), Spain \\ 3 Dept. of Astrophysics, Faculty of Physics, University of La Laguna, 38200 La Laguna (Tenerife), Spain \\ 4 Niels Bohr Institute, Julie Maries vej 30, 2100 Copenhagen Ø, Denmark
}

Received 30 June 2006 / Accepted 15 September 2006

\begin{abstract}
Aims. We investigate the effects of varying the magnetic field strength and the twist of a flux tube as it rises through the solar interior and emerges into the atmosphere.

Methods. Using a 3D numerical MHD code, we consider a simple stratified model, comprising of one solar interior layer and three overlying atmospheric layers. We set a horizontal, twisted flux tube in the lowest layer. The specific balance of forces chosen results in the tube being fully buoyant and the temperature is decreased in the ends of the tube to encourage the formation of an $\Omega$-shape along the tube's length. We vary the magnetic field strength and twist independently of each other so as to give clear results of the individual effects of each parameter.

Results. We find a self-similar evolution in the rise and emergence of the flux tube when the magnetic field strength of the tube is modified. During the rise through the solar interior, the height of the crest and axis, the velocity of the crest and axis, and the decrease in the magnetic field strength of the axis of the tube are directly dependent upon the initial magnetic field strength given to the tube. No such self-similarity is evident when the twist of the flux tube is changed, due to the complex interaction of the tension force on the rise of the tube. For low magnetic field strength and twist values, we find that the tube cannot fully emerge into the atmosphere once it reaches the top of the interior since the buoyancy instability criterion cannot be fulfilled. For those tubes that do advance into the atmosphere, when the magnetic field strength has been modified, we find further self-similar behaviour in the amount of tube flux transported into the atmosphere. For the tubes that do emerge, the variation in the twist results in the buoyancy instability, and subsequent emergence, occurring at different locations along the tube's length.
\end{abstract}

Key words. magnetohydrodynamics (MHD) - Sun: magnetic fields - Sun: interior - Sun: corona - methods: numerical Sun: atmosphere

\section{Introduction}

The active regions of the Sun are sites of large-scale flux emerging from the convection zone, characterised by sunspots on the photosphere. The introduction of new magnetic field from beneath the photosphere can be responsible for the destabilisation of existing structures and consequently induce large-scale dynamic coronal events, such as CMEs and flares.

It is widely accepted that sunspots are the product of $\Omega$-shaped flux tubes, which have risen from the base of the convection zone and broken through the photospheric surface. A horizontal, magnetic flux tube in both pressure balance and thermal equilibrium with its surroundings must be less dense than the external plasma and, therefore, will be buoyant. By this mechanism, a flux tube can rise through the convection zone and, due to a secondary buoyancy instability, enter the overlying atmosphere. It is believed that the bipolar sunspots mark the two locations were the tube axis intersects with the photosphere.

With the rapid advance of computing resources it has become possible to simulate the rise of flux tubes in the upper convection zone and their emergence into the atmosphere, both in $2 \mathrm{D}$ and $3 \mathrm{D}$. These numerical experiments have identified that a flux tube rising through the convection zone must be twisted if it is to retain most of its flux during the rise (Moreno-Insertis \& Emonet 1996; Dorch \& Nordlund 1998). Flux can then escape from the top of the convection zone into the atmosphere via buoyancy instabilities (Matsumoto \& Shibata 1992; Kusano et al. 1998). Once into the atmosphere a flux tube will experience rapid horizontal expansion due to the rapid reduction of the atmospheric gas pressure and a more subdued vertical expansion phase, which eventually slows (Matsumoto et al. 1993; Magara 2001; Fan 2001; Magara \& Longcope 2001). However, the vertical expansion encourages plasma to drain from the highest parts of the tube and it is then possible for the tube to undergo a secondary runaway vertical expansion (Miyagoshi \& Yokoyama 2004; Archontis et al. 2004; Manchester et al. 2004). Under certain conditions, the expanded tube will interact with the atmospheric magnetic field.

Comparison of simulation results with observations is necessary to ensure the effects seen in experiments are indeed physical. Leka et al. (1996) concluded, by tracking the movement of sunspots on the photosphere, that emerging flux tubes must be twisted prior to entering the atmosphere. The images by TRACE, in the $171 \AA$ channel, show coronal loops originating from two 
concentrated regions on the photospheric plane with the tops of the loops spread out in an arcade fashion. Similar images have been produced by the numerical simulations of Archontis et al. (2004).

The observational study by Ryutova \& Shine (2006) shows clear coupling between photospheric motions caused by the emerging flux process and changes in the structure of the corona. Thus, it is important to fully understand the emergence process so as to have a more detailed understanding of this delicate coupling.

Most simulations of buoyant, twisted, flux tubes use a constant twist magnetic field for the flux tube. A large collection of papers actually use the same equations to define the magnetic field of the tube. In the cylindrical coordinate system, $(\hat{\boldsymbol{r}}, \hat{\boldsymbol{\theta}}, \hat{\boldsymbol{y}})$, the magnetic field is given by $\boldsymbol{B}=\left(B_{r}, B_{\theta}, B_{y}\right)$, where

$$
\begin{aligned}
& B_{r}=0, \\
& B_{\theta}=\alpha r B_{y}, \\
& B_{y}=B_{0} \mathrm{e}^{-r^{2} / R^{2}} .
\end{aligned}
$$

A fuller discussion of these equations and the parameters is contained within Sect. 2.4. For now we only note that variation in the values of $B_{0}, \alpha$ and $R$ will vary the magnetic field strength of the tube, the twist of the field lines about the tube's axis and the radius of the tube, respectively. These equations also ensure that the twist associated with the tube is initially constant for all radii.

We have identified thirteen papers that use exactly this field definition and a further three papers that modify the transverse field distribution (2) and so have a non-constant twist profile with radius. Each paper considers different combinations of values within the parameter space for $B_{0}, \alpha$ and $R$. To demonstrate how our study fits with and differs from those already carried out, we briefly outline the contents of these specific papers.

Fan et al. (1998) consider the rise of a highly twisted and, therefore, kink-unstable flux tube within the convection zone while Dorch et al. (2001) compares the rise of a tube in convective and non-convective flows. Moreno-Insertis \& Emonet (1996), Emonet \& Moreno-Insertis (1998), Abbett et al. (2000), Cheung et al. (2006) and Dorch (2003) vary the degree of twist of a tube rising though the convection zone. The first four papers aim to understand the fragmentation of the flux tube during its rise whilst the fifth considers the various instabilities arising from the different amounts of twist. The effects of variations in the magnetic field strength and twist of a tube rising though a convective flow are identified by Fan et al. (2003). Dorch et al. (1999) compares the rise of horizontal and undular twisted flux ropes in both $2 \mathrm{D}$ and $3 \mathrm{D}$ during their rise through the convection zone.

The remaining papers consider the full emergence of the flux tube from the solar interior into the overlying atmosphere. Abbett \& Fisher (2003), using two different computational codes to simulate the full emergence process, find that decreasing the twist of the flux tube increases the degree to which the initial emergence is force-free. Fan (2001) considers a tube which is stable to the kink-instability and reports on the dynamic evolution of the tube during the emergence process into an unmagnetised atmosphere. A whole series of further studies have sprung from the results of this single experiment. A variety of values for the field strength, twist and radius are used but each subsequent study considered just one value for each parameter.

Archontis et al. (2004) have further investigated the results of Fan (2001), reporting them in more detail and advancing the experiment by adding a magnetised atmosphere above the emerging flux tube. Galsgaard et al. (2005) and Archontis et al. (2005) have varied the structure of the magnetised atmosphere and use the same parameter values as Archontis et al. (2004). Manchester et al. (2004) use the same parameter values as the original experiment by Fan (2001) but they reduce the region over which the tube is buoyant and find a CME type event occurs once the tube has emerged into the atmosphere. Finally, Leake \& Arber (2006) include a partially ionised region in the atmosphere into which the flux tube emerges.

The extensive use of the field structure given by (1)-(3), in emergence experiments warrants a comprehensive study of the parameter space. We vary the twist and strength of a magnetic flux tube and, for the first time in an emergence study, evaluate the effects of the variations. Our aim is to understand the role of twist and field strength in shaping the emergence process.

It should be noted that a parameter space investigation has been carried out by Magara (2001), in which the radius, twist and strength of an emerging flux tube were varied. Our study is different on several fronts. We use the field structure defined by (1)-(3), and this is not used by Magara (2001). The flux tube we define is fully buoyant and, therefore, prescribed with a buoyancy perturbation (described in Sect. 2.4) to encourage the formation of an $\Omega$-shaped loop. Following this initialised perturbation we allow the system to evolve by itself. On the other hand, Magara (2001) choose to drive the central portion of the tube upwards by imposing a vertical fluid velocity. Finally, the values of the parameters were altered simultaneously by Magara (2001) and, therefore, it is difficult to quantify the independent impact of each on the emergence process. We aim to provide a clear explanation of how varying the twist and field strength of the flux tube, independently of each other, will alter the dynamics of the rise of the flux tube in the upper layers of the convection zone and its subsequent expansion into the atmosphere. A study by Miyagoshi et al. (2006) investigates the effects of varying the twist of an emerging flux tube with a different magnetic field profile to that considered within this paper.

The paper is laid out as follows: Sect. 2 describes the model and numerical code. Section 3 presents the results of varying the magnetic field strength (3.1) and twist (3.2) of the flux tube. Section 4 contains a summary of the results and the main conclusions.

\section{Model setup}

In this series of experiments, we follow the setup used in Archontis et al. (2004), in which a flux tube emerges from the solar interior into an unmagnetised atmosphere. The stratification of the atmosphere, the magnetic profile of the tube and the form of the density deficit along the tube's axis that are used in the simulations by Archontis et al. (2004) are based upon those originally used by Fan (2001).

\subsection{Equations}

For the experiments in this paper, we solve the threedimensional, time-dependent, resistive MHD equations numerically. These equations are:

$$
\begin{aligned}
\frac{\partial \rho}{\partial t} & =-\nabla \cdot(\rho \boldsymbol{v}), \\
\frac{\partial(\rho \boldsymbol{v})}{\partial t} & =-\nabla \cdot(\rho \boldsymbol{v} \otimes \boldsymbol{v}+\hat{\tau})-\nabla p+\rho \boldsymbol{g}+\boldsymbol{J} \times \boldsymbol{B},
\end{aligned}
$$




$$
\begin{aligned}
\frac{\partial e}{\partial t} & =-\nabla \cdot(e \boldsymbol{v})-p \nabla \cdot \boldsymbol{v}+Q_{\text {Joule }}+Q_{\text {visc }}, \\
\frac{\partial \boldsymbol{B}}{\partial t} & =-\nabla \times \boldsymbol{E}, \\
\boldsymbol{E} & =-\boldsymbol{v} \times \boldsymbol{B}+\frac{\boldsymbol{J}}{\sigma}, \\
\boldsymbol{J} & =\nabla \times \frac{\boldsymbol{B}}{\mu} \\
p & =\rho T \frac{\mathcal{R}}{\tilde{\mu}},
\end{aligned}
$$

with density $\rho$, velocity $\boldsymbol{v}$, viscous stress tensor $\hat{\tau}$, gas pressure $p$, acceleration due to gravity $\boldsymbol{g}$, electric current density $\boldsymbol{J}$, magnetic field $\boldsymbol{B}$, thermal energy per unit volume $e=p /(\gamma-1)$, Joule dissipation $Q_{\text {Joule }}$, viscous dissipation $Q_{\text {visc }}$, electrical resistivity $\sigma$, magnetic permeability $\mu$, temperature $T$, gas constant $\mathcal{R}$, average atomic mass per particle $\tilde{\mu}$, respectively. $\gamma$ is the ratio of specific heats and is taken to be $5 / 3$.

\subsection{Numerical code}

We use a 3-dimensional code developed by Nordlund \& Galsgaard (1997). The MHD equations are solved on staggered grids using a sixth order accurate method to evaluate spatial derivatives and a fifth order interpolation method for shifting variables between the different grids. An explicit third order predictor-corrector method is used to advance the solution in time.

A grid of $148 \times 160 \times 218$ points is used to model an integration box with a dimensionless domain $(-60,60) \times(-70,70) \times$ $(-22,70)$ in the $(x, y, z)$ co-ordinate system. This corresponds to a domain of $20.4 \mathrm{Mm} \times 23.8 \mathrm{Mm} \times 15.6 \mathrm{Mm}$ on the Sun. The grid has uniform spacing in the horizontal plane but is stretched in the vertical direction. The numerical resolution is highest over the region from just below the initial location of the flux tube to just above the transition region. The gridspacing represents a physical distance of $4.77 \times 10^{4} \mathrm{~m}$ at its smallest and $2.49 \times 10^{5} \mathrm{~m}$ at its largest.

The boundaries of the box are periodic in the horizontal directions and closed on the top and bottom. A damping region is included at both the top and bottom of the box to limit the reflection of waves.

\subsection{Background stratification}

We define a background stratification to model four distinct regions of the Sun, namely the upper layers of the solar interior, the photosphere, transition region and corona. The starting height of the photosphere, transition region and corona, in dimensionless form, are $z_{\mathrm{ph}}=0, z_{\mathrm{tr}}=10$ and $z_{\mathrm{c}}=20$ respectively.

The solar interior is chosen to have a linearly increasing temperature profile with depth and constant entropy, making the region neutrally stable to the onset of convection. The photosphere and corona are isothermal, with the corona being 150 times hotter than the photosphere, $T_{\mathrm{c}}=150 T_{\mathrm{ph}}$. The temperature in the transition region is given by

$T(z)=T_{\mathrm{ph}}\left(\frac{T_{\mathrm{c}}}{T_{\mathrm{ph}}}\right)^{\left(z-z_{\mathrm{tr}}\right) /\left(z_{\mathrm{c}}-z_{\mathrm{tr}}\right)}$.

All of the regions are in hydrostatic balance prior to the tube being placed in the solar interior.
Table 1. Summary of the parameter space under investigation.

\begin{tabular}{cc}
\hline \hline group 1 & group 2 \\
\hline$B_{0}=\{1.0,2.0,3.0,5.0,9.0\}$ & $B_{0}=2.9$ \\
$\alpha=0.4$ & $\alpha=\{0.1,0.2,0.3\}$ \\
\hline
\end{tabular}

We choose the non-dimensional temperature, density, and pressure scale-height to be $T_{\mathrm{ph}}=1, \rho_{\mathrm{ph}}=1, H_{\mathrm{ph}}=1$, respectively, at the base of the photosphere. Physical units are obtained by multiplying the dimensionless quantities by $T_{\mathrm{ph}}=5.6 \times 10^{3} \mathrm{~K}$, $\rho_{\mathrm{ph}}=3 \times 10^{-4} \mathrm{~kg} \mathrm{~m}^{-3}, H_{\mathrm{ph}}=1.7 \times 10^{5} \mathrm{~m}$. From these values we define a photospheric gas pressure unit $p_{\mathrm{ph}}=1.4 \times 10^{11} \mathrm{erg} \mathrm{m}^{-3}$, a velocity unit $V \equiv\left(p_{\mathrm{ph}} / \rho_{\mathrm{ph}}\right)^{1 / 2}=6.8 \times 10^{3} \mathrm{~m} \mathrm{~s}^{-1}$, a time unit $t_{\mathrm{ph}}=25 \mathrm{~s}$ and a magnetic field unit $B_{\mathrm{ph}}=1.3 \times 10^{3}$ Gauss. Surface gravity is $2.7 \times 10^{2} \mathrm{~m} \mathrm{~s}^{-1}$ and $\tilde{\mu}=1$.

\subsection{Magnetic flux tube}

We insert a twisted, magnetic flux tube that is lying horizontally near the top of the solar interior. As discussed in Sect. 1, we define the magnetic field of the tube in cylindrical co-ordinates using (1)-(3). The axis of the tube is initially located at $z=-10$ with the top of the solar interior at $z=0$. The background plasma in the solar interior is unmagnetised and, thus, we require the magnitude of the tube's magnetic field to be zero at large radial distances from the axis. The exponential functions in $r^{2}$, contained within each of the tube's nonzero magnetic components, ensure the field strength decreases with radial distance from the axis following a Gaussian profile. The magnetic field on the axis has solely a $B_{y}$ component, with direction given by the sign of $B_{0}$ and strength by $\left|B_{0}\right|$. At a distance $R$ from the axis, the component of the field in the axial direction is $37 \%$ its original value. The magnetic field lines of the tube twist around its axis, where the twist is defined as

$\Phi=\frac{B_{\theta}}{r B_{y}}=\alpha$.

Hence, independent of the radial distance from the axis, each field line must rotate about the axis through an angle of $\alpha$ radians over a distance of 1 axial unit.

The value of $R$ is held fixed at 2.5 over the series of experiments and $B_{0}$ and $\alpha$ are varied. The experiments are split into two groups, group 1 for those with fixed $\alpha$ and varying $B_{0}$ and group 2 for fixed $B_{0}$ with varying $\alpha$. A summary of the values of $B_{0}$ and $\alpha$ under consideration is given in Table $1 . \alpha$ is constant with radius in each of the experiments so that the tubes are uniformly twisted. Additionally, the group 1 cases, with $\alpha$ fixed at 0.4 , are marginally unstable to the kink instability since $\alpha>1 / R$ (Fan et al. 1998), however, we do not see the instability develop during the tube's rise through the solar interior in any of our experiments.

We require the tube to be in radial force balance with the external plasma. The internal gas pressure of the tube, $p_{\mathrm{i}}$, is defined as $p_{\mathrm{e}}+p_{\mathrm{exc}}$ where $p_{\mathrm{e}}$ is the external gas pressure and $p_{\mathrm{exc}}$ is the pressure excess. Given the field prescribed by (1)-(3), we therefore require

$p_{\text {exc }}=\frac{1}{2 \mu}\left(\alpha^{2}\left(R^{2} / 2-r^{2}\right)-1\right) B_{y}^{2}$,

for pressure balance.

As with the gas pressure, we define the density of the material in the tube, $\rho_{\mathrm{i}}$, to be $\rho_{\mathrm{e}}+\rho_{\mathrm{exc}}$ where $\rho_{\mathrm{e}}$ is the external density 
and $\rho_{\mathrm{exc}}$ is the density excess. If we were to choose the whole of the tube to be in thermal equilibrium with its surroundings then the density excess would be given by

$\rho_{\mathrm{exc}}=\frac{\tilde{\mu} p_{\mathrm{exc}}}{\mathfrak{R} T(z)}=\frac{\tilde{\mu}}{2 \mu \mathfrak{R} T(z)}\left(\alpha^{2}\left(R^{2} / 2-r^{2}\right)-1\right) B_{y}{ }^{2}$.

The whole tube would then be buoyant when a density deficit exists, $\rho_{\text {exc }}<0$. Interpreted physically, this means that the outwardly acting magnetic pressure gradient is larger than the inwardly acting tension force and therefore the gas pressure gradient also acts inwardly. This criterion for buoyancy is independent of $B_{0}$ and is satisfied at all radii when $|\alpha| \leq 0.57$. Thus all of the tubes within groups 1 and 2 would have a density deficit and, therefore, they would be buoyant at all radii since the twist of each satisfies $|\alpha| \leq 0.57$.

However, we choose only the cross-section of the tube at $y=0$ to have this complete buoyancy at all radii. To encourage the formation of an $\Omega$-loop shape along the tube's length the density deficit is reduced away from $y=0$ following the Gaussian profile

$\rho_{\mathrm{m}}=\rho_{\mathrm{exc}} \mathrm{e}^{-y^{2} / \lambda^{2}}$,

where $\lambda=20$, such that the density in the tube is now given by $\rho_{\mathrm{i}}=\rho_{e}+\rho_{\mathrm{m}}$. The smaller density deficit for $|y|>0$ implies that there is a corresponding increase in the temperature contrast between the tube and surroundings.

From (14) we can see that both the twist and the magnetic field strength play a role in determining how buoyant the flux tube will be. For group 2, the variation in $\alpha$ leaves the strength of the field at the tube's axis unchanged and has little effect on the intensity of the magnetic field at outer radii, as shown in Fig. 1a. However, increasing the amount of twist will increase the inwardly acting tension force more than the outwardly acting magnetic pressure force and, therefore, will alter the buoyancy of the tube. Thus the tubes in group 2 are buoyant to varying degrees despite the small variation in their overall magnetic field intensity.

From Fig. 1b it can be seen that for the cases in group 1, there is a large variation in $B_{0}$, and, consequently, there will be a large variation in the initial buoyancy of the tube, both at the axis and at all radii until the edge of the tube is reached. Since, from (14), the density deficit is proportional to $B_{0}^{2}$, the tube with $B_{0}=9.0$ is 9 times more buoyant than that with $B_{0}=3.0$, which in turn is 9 times more buoyant than the tube with $B_{0}=1.0$.

\section{Results}

For the tube with $B_{0}=3.0$ and $\alpha=0.4$, from group 1, we see the same results as those described in Archontis et al. (2004). As the buoyant portion of the tube rises it compresses the plasma preceding it, which is rising more slowly. This compression causes the transverse magnetic field to be enhanced at the leading edge of the tube, thus the field lines turn further from the axial direction.

As the tube rises, it moves to heights where the external gas pressure is continually decreasing. The tube tries to maintain horizontal force balance and so expands. As a consequence of the expansion, there is a fall in the internal density but the tube becomes increasingly less buoyant in comparison with its surroundings. Eventually, parts of the tube become over dense whilst the tube is still in the solar interior. However, the tube continues to rise as a whole. By mass conservation and magnetic flux conservation, the magnetic field strength in the central (a)

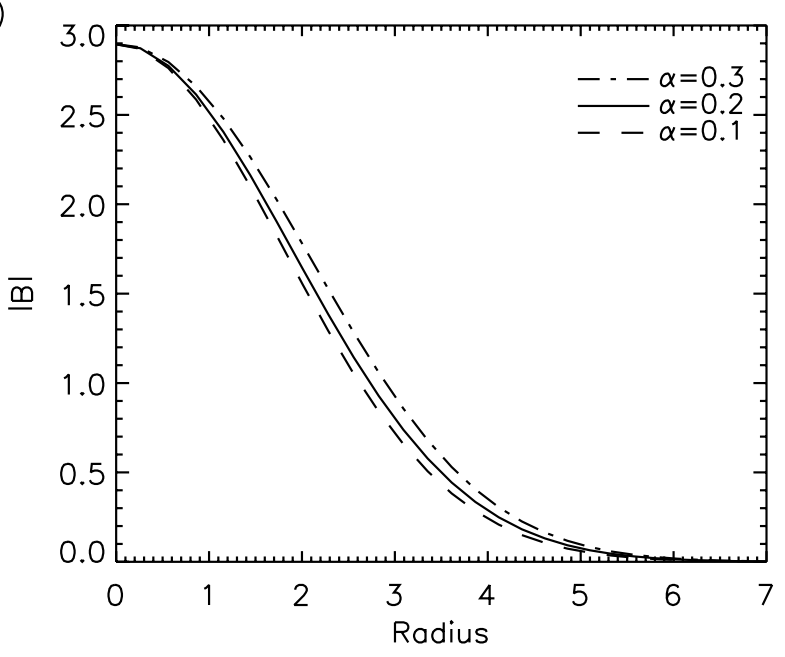

(b)

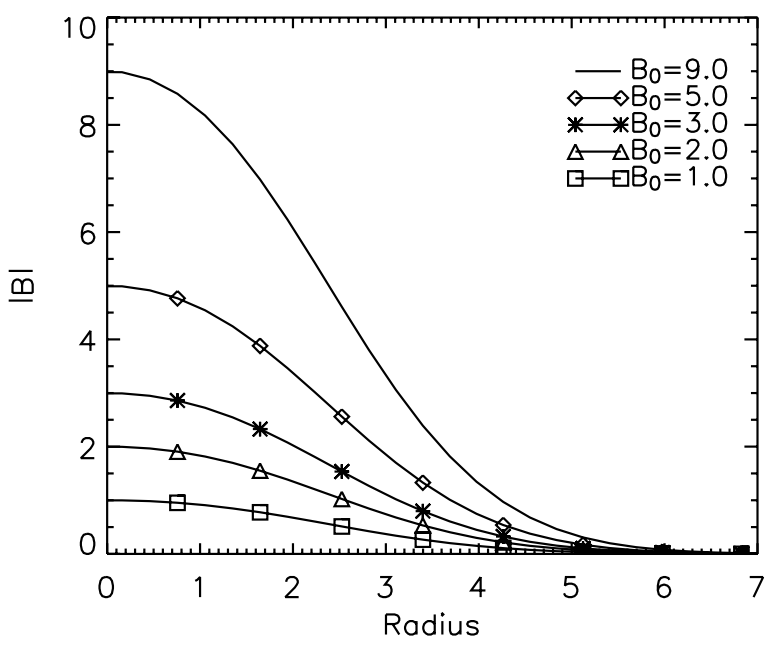

Fig. 1. Radial distribution of the initial magnetic field strength, $|\boldsymbol{B}|$, for cases with varying a) $\alpha$ and b) $B_{0}$.

part of the tube decreases due to the expansion of the tube during the rise.

The temperature in the photosphere is isothermal, hence the stratification is strongly subadiabatic and, following the classical Schwarzschild criterion, is stable against convection. Thus, the tube is no longer buoyant compared with its surroundings when it reaches the photosphere. Through the development of a magnetic buoyancy instability, the tube can rise into the atmosphere where it undergoes a marked expansion due to the sharp fall in background gas pressure with height. The axis of the tube reaches equilibrium at the photosphere and does not rise into the atmosphere within the limited time of the experiment.

We now consider how varying $B_{0}$ and $\alpha$ modifies the rise of the tube through the solar interior and its emergence into the atmosphere.

\subsection{Group 1: varying $B_{0}$ with fixed $\alpha$}

\subsubsection{Rise in the solar interior}

All of the tubes are buoyant and initially at rest, thus the motion of the tubes at the start of the experiments is characterised by a vertical velocity. From (14), we know that the buoyancy force in 
(a)

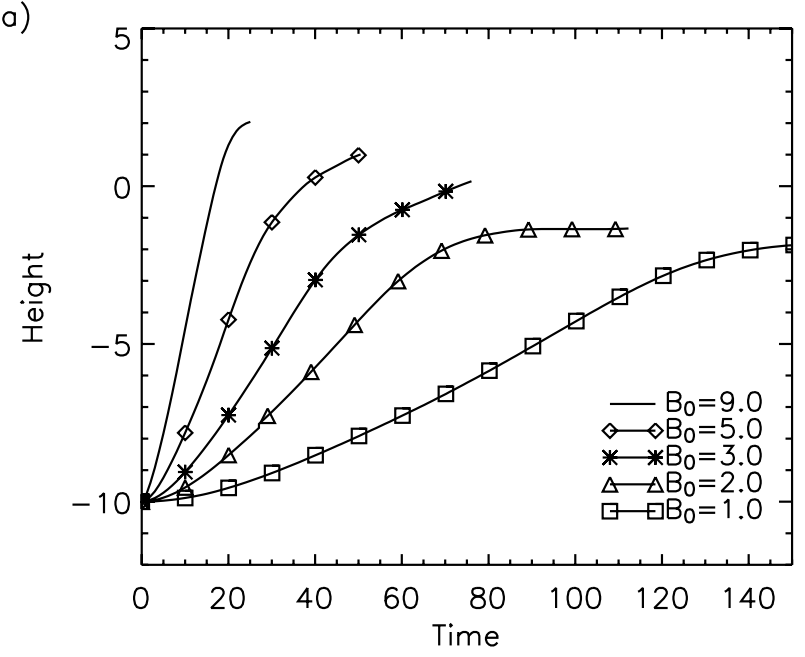

(b)

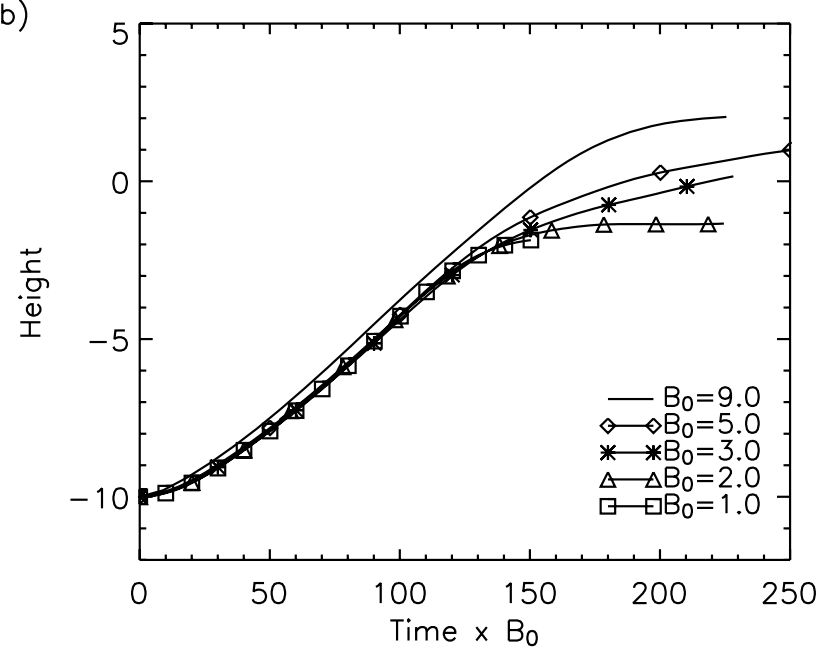

Fig. 2. Height of the axis of the tube for the various $B_{0}$ cases, measured over a) time, $t$, and b) rescaled time, $\bar{t}=t B_{0}$.

the central portion of the tube is proportional to $B_{0}^{2}$ and therefore expect tubes with larger values of $B_{0}$ to have higher velocities and reach greater heights more quickly. This is confirmed by Fig. 2a, which shows that the time taken for the axis of the tube to reach $z=-2$, a height just below the photosphere, in each of the cases is inversely proportional to $B_{0}$.

By redefining time as $\bar{t}=t B_{0}$ and rescaling the horizontal axis in Fig. $2 \mathrm{a}$ we find that the heights reached by the axes are similar to each other up until a time of approximately $\bar{t}=150$ (see Fig. 2b). Rescaling time in this way is equivalent to measuring time on an Alfvén timescale rather than a sound timescale. A similar reliance on the initial field strength is found for the rise of the crests of the tubes through the solar interior. Thus the heights of the axis and crest of each of the tubes is not only a function of time but also initial field strength, i.e. $H(\bar{t})=H\left(t B_{0}\right)$ where $H$ is the function giving the height of the axis or crest of the tube. This inverse scaling of the rise times of the tubes with $B_{0}$ in unconvective flows confirms the $2 \mathrm{D}$ results previously found by Fan et al. (2003).

The initial acceleration of the tube is proportional to the buoyancy force acting on the tube and, therefore, the vertical acceleration will be proportional to $B_{0}^{2}$ for the group 1 cases. (a)

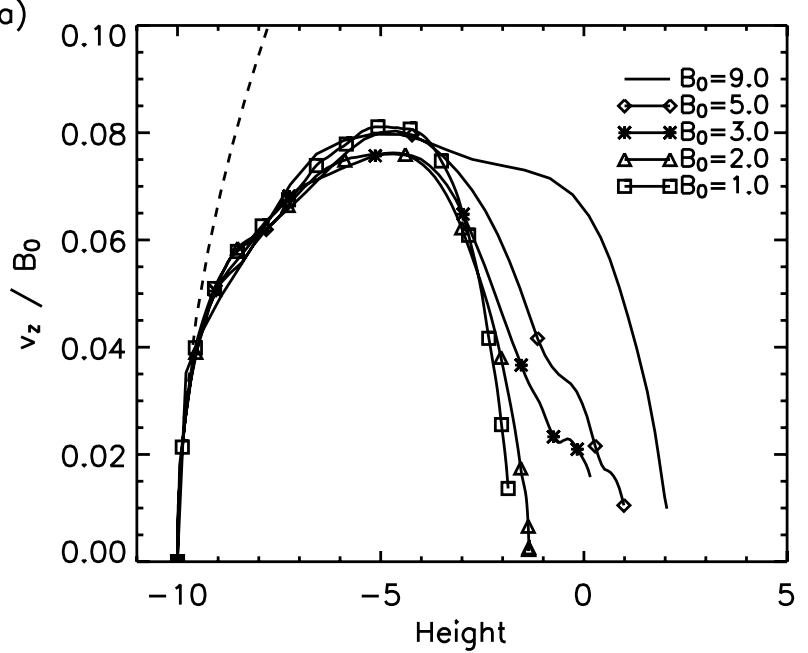

(b)

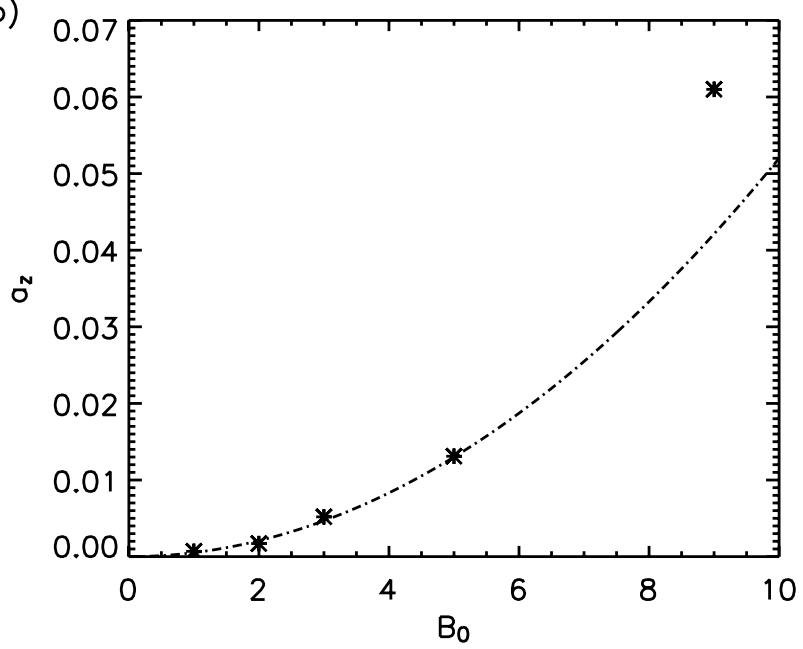

Fig. 3. Measures of the rate of rise of the axis of the tube for the various $B_{0}$ cases: a) the vertical velocity as a fraction of the initial field strength of the axis measured against the height of the axis, and b) the constant vertical acceleration $\left(a_{z}\right)$ during the second period. The dashed line in a) is the vertical acceleration expected from free-fall alone and is given by integrating (16). The dot-dashed line in $\mathbf{b}$ ) is given by $a_{z}=0.0005 B_{0}^{2}$ and identifies the acceleration of the tube as proportional to $B_{0}^{2}$.

To make comparisons between the tubes when they are at the same heights we redefine time to be $\bar{t}$ using the scaling described above. Thus, the vertical velocity as a function of height will be proportional to $B_{0}$, as verified by Fig. 3a. Interestingly, the 2D study by Emonet \& Moreno-Insertis (1998) found that variations in the initial plasma- $\beta$ of the tube resulted in similar velocity profiles once the velocity scale was enlarged by a factor $\beta_{0}^{1 / 2}$ and the time scale was contracted by the same factor. These scalings by $\beta_{0}^{1 / 2}$ are exceedingly similar to the scalings used within this paper. Application of the $\beta_{0}^{1 / 2}$ scalings to our data reveals that the scalings we use provide greater similarity between the velocity profiles of our tubes during the acceleration phase.

Previous studies (Moreno-Insertis \& Emonet 1996; Emonet \& Moreno-Insertis 1998) distinguish different phases in the acceleration of a buoyant flux tube rising in a stratified medium. At the very beginning there is a free-fall period in which the acceleration of the tube is purely caused by its own buoyancy. 
Emonet \& Moreno-Insertis (1998) give this free-fall acceleration at the tube's axis as

$a_{z}=\frac{g}{i}\left|\frac{\Delta \rho}{\rho}\right|_{r=0}$,

where $i=2$ is the enhanced inertia factor due to the co-moving surrounding medium, $\Delta \rho$ is the density excess, $\rho_{\text {exc }}$, and $\rho$ is the internal density. Both $\Delta \rho$ and $\rho$ are measured at the axis at time $t=0$. Integrating (16) gives the vertical velocity, which is plotted as the dashed line in Fig. 3a and agrees relatively well with the vertical velocity of the tube's axis for the group 1 cases.

In the second phase, which starts when the axis is only a short distance from its initial height, Moreno-Insertis \& Emonet (1996) and Emonet \& Moreno-Insertis (1998) state that the acceleration diminishes due to the increasingly important aerodynamic drag exerted by the flows surrounding the tube. In fact, between the heights of $z \sim-9$ and $z \sim-6$ we find that there is a period of roughly constant, slower acceleration. As expected from the overlying of the curves in Fig. 3a, during this period the vertical acceleration of axes of the tubes remains proportional to $B_{0}^{2}$, as illustrated by Fig. $3 \mathrm{~b}$. Once this period of constant acceleration ceases, the acceleration continues to diminish as the tube rises higher.

The final, asymptotic phase, described in the papers mentioned above, represents the period when the buoyancy force and the aerodynamic drag balance each other, giving rise to a continued period of average zero acceleration. In our calculation, this asymptotic phase cannot be reached, since by then the tube is close to the photosphere and the acceleration of the tube is determined by additional factors, described in the following section, not present in the previous studies. In fact, in our case, when the axes of the tubes are at $z \sim-5$, the acceleration is momentarily zero before becoming negative. At the zero acceleration stage, the vertical magnetic forces only contribute $\sim 5 \%$ towards the total unsigned force $\left(\boldsymbol{F}_{z}=|-\nabla p|_{z}+|\rho \boldsymbol{g}|+|\boldsymbol{J} \times \boldsymbol{B}|_{z}\right)$ acting on the axis. Hence, the zero vertical acceleration at the axis occurs when the vertical gas pressure gradient approximately balances the buoyancy force. The decrease in the vertical velocity of the axis occurs when the plasma there is still buoyant but the gradient in the gas pressure is the largest force.

Previous papers dealing with the 2D case (Moreno-Insertis \& Emonet 1996; Emonet \& Moreno-Insertis 1998; Cheung et al. 2006) have reported oscillations in the vertical velocity of the axis and crest of the tube. These oscillations are part of the internal torsional oscillations taking place in the interior of the rising tube (Emonet \& Moreno-Insertis 1998); they decrease in amplitude as the tube rises: the energy loss is due to transmission to the surroundings of the tube via pressure forces and to internal diffusion. We do not see marked oscillations in our experiments. This may be due to a combination of factors. Firstly, our tubes are substantially thicker (compared to the local stratification scaleheight) than those of the previous calculations. The ratio of the oscillation period to the risetime across one scaleheight is therefore longer in our case. Secondly, our 3D case has lower numerical resolution and, hence, probably also higher numerical diffusion. Thirdly, in the 2D case, the whole length of the tube along its axis was oscillating with the same amplitude and in phase. This is no longer the case in the 3D experiment; the oscillation of the rising section of the tube is transmitted to the lower, non-rising stretches, and, therefore, the amplitude becomes smaller.

Also of interest are changes to the gas pressure and density profiles within the tube. The initial density deficit $\left(\rho_{\mathrm{exc}}<0\right)$ and

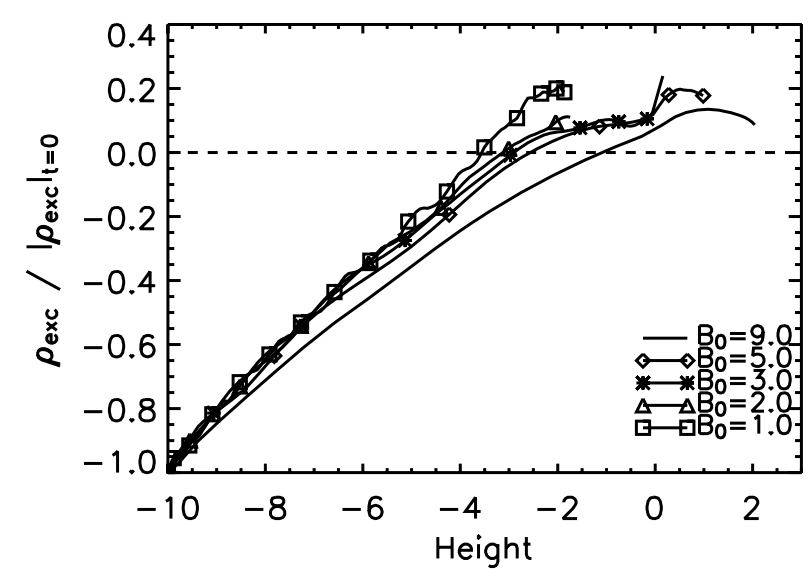

Fig. 4. The increase in the density excess at the axis, as a fraction of the initial unsigned density excess there, plotted against the height of the axis for the various $B_{0}$ cases.

gas pressure deficit $\left(p_{\text {exc }}<0\right)$ in each tube are small in comparison to the external density and gas pressure. Thus, the internal density and gas pressure are dominated by their external values. As the tube rises, we find that this dominance is maintained and we see the density and gas pressure of the rising axis behaving as the external quantities at the associated height. Hence, the density and the gas pressure at the axis of the tube are given by

$\rho_{\mathrm{i}}\left(z_{a}\right) \propto\left(1-2 z_{a} / 5\right)^{3 / 2}$,

$p_{\mathrm{i}}\left(z_{a}\right) \propto\left(1-2 z_{a} / 5\right)^{5 / 2}$,

where $z_{a}$ is the height of the axis and $z_{a}<0$.

Figure 4 shows the density excess at the axis, given as a fraction of the initial unsigned density excess there, plotted against the height of the axis. It can be seen that the axes of the tubes becomes over dense $\left(\rho_{\text {exc }}>0\right)$ prior to them reaching the base of the photosphere. This indicates that, for each tube in group 1 , all of the material above the axis of the tube also loses its buoyancy before reaching the photosphere. The density excess as a proportion of the actual internal density is small and, therefore, its growth is marginal in comparison to the overall fall in the internal density.

Figure 5 considers the change in the field strength at the axis of each tube, as a fraction of its initial field strength, and shows a similar pattern of decrease as each tube rises. The thin flux tube approximation states that $|\boldsymbol{B}| / \rho$ should be conserved but this approximation is only valid when the radius of the tube is negligible compared with the scale height of the surrounding unmagnetised fluid and any scales of variation along the tube (see the review by Fan (2004) and references therein for a complete discussion). The flux tubes we consider have a radius that is 2.5 times the photospheric pressure scale height and are twisted so this approximation is not valid for the whole of the tube. However, since the axis of the tube is untwisted and the most buoyant section at $(x=0, y=0)$ remains horizontal for all time, we find that

$\left|B_{y}\left(z_{a}\right)\right|=\frac{\left|B_{0}\right|}{\rho_{0}}\left(1-2 z_{a} / 5\right)^{3 / 2}$,

where $\rho_{0}$ is the density measured at the axis at $t=0$, is in fact a good approximation.

The decrease shown in Fig. 5 is well prescribed by (19) (dashed line in Fig. 5) until the axes reach $z \sim-7$, a sizeable 


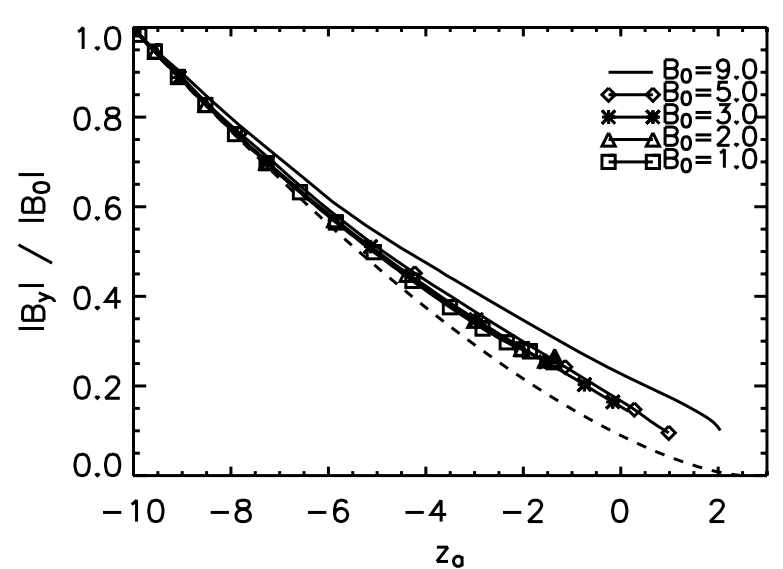

Fig. 5. The decrease in the $B_{y}$ component of the field at the axis, as a fraction of the initial field strength there, measured at $(x=0, y=0)$ and plotted against the height of the axis, $z_{a}$. The dashed line corresponds to the decrease in the axial magnetic field component, $B_{y}$, at the axis in accordance with the thin flux tube approximation given by (19).

proportion of the total distance travelled by each axis. There is an $8 \%$ and a $21 \%$ deviation of the approximation from the simulation values when the axes reach heights of $z_{a}=-5$ and $z_{a}=-2$, respectively. This deviation occurs because the differential buoyancy along the axis of the tube causes it to adopt an $\Omega$-shape along its length. The bending of the axis encourages draining of plasma and, therefore, the density at the axis decreases at a faster rate than solely through rising.

The discussion within this section has seen the rescaling of several quantities with respect to the only varied parameter of the model, $B_{0}$. By rescaling, we have been able to remove the dependence of $B_{0}$ from the model, revealing a self-similar evolution of the tubes during their rise through the solar interior. As we will see, this self-similarity is also visible when the tubes enter the photosphere and in those that emerge into the atmosphere.

\subsubsection{Emergence into the photosphere}

As discussed in Sect. 3.1.1, when the top of the tube rises through the last few remaining pressure scale-heights of the solar interior it is over dense in comparison with the surrounding medium but continues to rise due to the external adiabatic stratification. However, the photosphere is strongly subadiabatic and, therefore, there is a rapid slowing of the rise of the crest of the tube when it enters the lower atmosphere.

The plasma in the upper part of the tube cannot continue to rise vertically due to the slowing of the crest and, it is forced to spread out horizontally at the photosphere to make way for the rising material below (Magara 2001; Archontis et al. 2004). The area of the interface between the solar interior and the photosphere that contains flux from the tube steadily increases with time, see Fig. 6a. Tubes with a stronger initial field strength spread out and cover a larger photospheric area more quickly than those with a lower initial strength. Figure $6 \mathrm{~b}$ shows that the time taken for each of the tubes to cover the same sized area is proportional to $B_{0}$. This indicates that the faster spread of the higher strength tubes is purely due to their faster rise to the photosphere, which occurs due to their greater initial buoyancy force. Additionally, since only some of the tubes go on to fully emerge into the atmosphere (as will be discussed shortly)
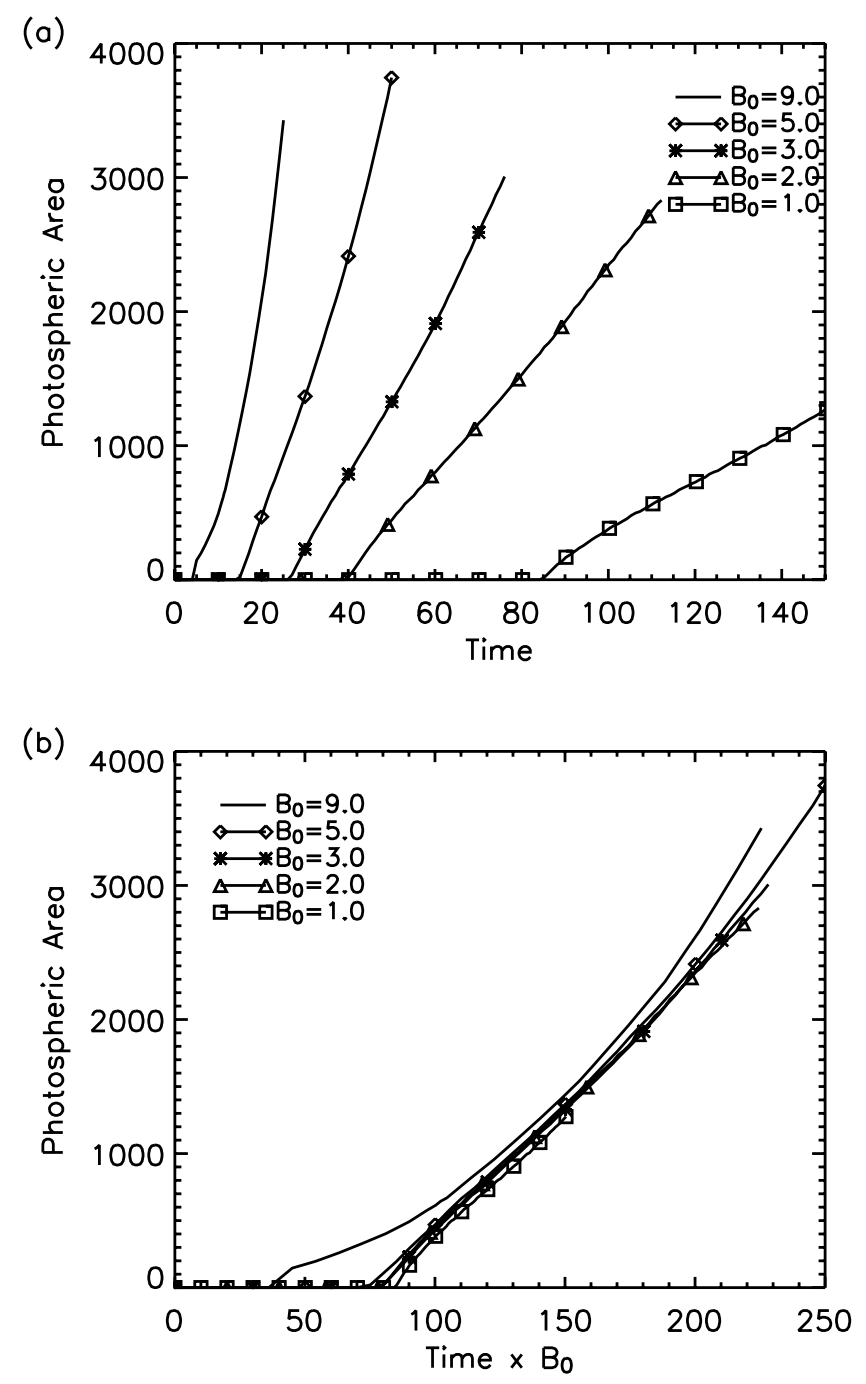

Fig. 6. Measurements of the dimensionless area of the photospheric plane $(z=0)$ containing flux from the emerging tube for the various $B_{0}$ cases compared over a) time, $t$, and b) rescaled time, $\bar{t}=t B_{0}$. The dimensionless length unit at the photosphere, $L_{\mathrm{ph}}$, is equivalent to $170 \mathrm{~km}$.

we know that this occupation of the photospheric plane is controled purely by the tube plasma rising below the photosphere and is independent of the transportation of tube flux into the upper atmosphere.

Many studies have shown that over-dense flux can be transported into the atmosphere via the magnetic buoyancy instability. Perturbations that bend the field lines cause the instability to be launched more quickly. Emergence into the upper atmosphere occurs when

$-H_{p} \frac{\partial}{\partial z}(\log B)>-\frac{\gamma}{2} \beta \delta+\tilde{k}_{\|}^{2}\left(1+\frac{\tilde{k}_{\perp}^{2}}{\tilde{k}_{z}^{2}}\right)$

is satisfied (Newcomb 1961; Yu 1965; Thomas \& Nye 1975; Acheson 1979). In this criterion we have the pressure scaleheight, $H_{\mathrm{p}}$, the ratio of specific heats, $\gamma$, the plasma- $\beta$ and perturbations with wavevector $\boldsymbol{k}$ (where $\tilde{k_{\|}}$and $\tilde{k_{\perp}}$ are the horizontal components parallel and perpendicular to the magnetic field and $\tilde{k}_{z}$ is the vertical component). The superadiabatic excess, $\delta$ is given by $\delta=\nabla-\nabla_{\mathrm{ad}}$, where $\nabla$ is the actual logarithmic temperature gradient in the equilibrium stratification and $\nabla_{\text {ad }}$ is its 
adiabatic value. The review by Moreno-Insertis (2005) considers the development of the terms in (20) prior and post instability development. Here, we investigate only the differences in the size of the terms of (20) between the various $B_{0}$ cases to understand why some tubes emerge whilst others do not.

Two of the tubes under consideration in group 1 do not emerge into the atmosphere and these are the ones with the lowest values of $B_{0}$, namely 1.0 and 2.0. Consideration of each of the terms in (20) clarifies why these tubes do not emerge. To make a comparison between the different cases we consider the lefthand and right-hand quantities of the criterion at the time when the criterion is first satisfied for each tube. This time is different for each of the three tubes that emerge and the times considered are $t=15,27$ and 49 for $B_{0}=9.0,5.0$ and 3.0, respectively. Since two of the tubes do not emerge, (20) is never satisfied in these cases so we choose times such that the tops of these tubes have advanced into the photosphere. For $B_{0}=2.0$ and $1.0 \mathrm{we}$ choose $t=70$ and 152 .

The term on the left-hand side of (20) corresponds to the compression of the magnetic field and, by taking the natural logarithm of the field, it is independent of $B_{0}$. Figure $7 \mathrm{f}$ shows the natural logarithm of the magnitude of the magnetic field, where the magnitude of the field has been made independent of $B_{0}$, for the various group 1 cases at the times stated above. We consider this quantity at the height at which the instability terms, as calculated for panels (a)-(e) of Fig. 7, are non-zero for each tube, namely $z=1$ and $z=2$ for the $B_{0}=1.0$ and 2.0 cases respectively, and $z=3$ for the remaining tubes. We can see that the tubes with the smaller values of $B_{0}$ have the steepest gradient at the given height. The reason for this is as follows:

When the crest of the tube enters the photosphere it is forced to slow due to the change in the background stratification. The tubes with higher values of $B_{0}$ have a higher velocity when rising through the solar interior and, therefore, they have a higher momentum when their front reaches the photosphere so the velocity of the tube front does not decrease significantly. Equivalently, the tubes with smaller values of $B_{0}$ have a smaller velocity and therefore a smaller momentum so their tube fronts slow greatly in the photosphere. The axes of all the tubes are still rising and in the cases of $B_{0}=1.0$ and $B_{0}=2.0$ the distance between the crest and the axis begins to decrease again since the tube fronts are no longer rising as quickly. The material at the front of these tubes is therefore compressed to a greater extent and this results in the gradient of the magnetic field strength steepening.

The first term on the right-hand side of (20) contains the plasma- $\beta$. The initial plasma- $\beta$ is significantly higher for those tubes with a smaller value of $B_{0}$. This is because these tubes have a larger internal gas pressure and a smaller magnetic pressure. Irrespective of $B_{0}$, as the tube rises the value of the plasma$\beta$ for the material in the front portion of the tube decreases but we note that the tubes with smaller values of $B_{0}$ still have the largest plasma- $\beta$ since the magnitude of their magnetic field is smaller.

In Figs. 7a-e we show the left-hand and right-hand terms of (20), divided by the plasma- $\beta$, at the times previously specified for each tube.

From the discussion above, we know that the gradient in the magnetic field profile is smaller for higher valued $B_{0}$ tubes, with the left-hand term for the $B_{0}=9$ tube being $\sim 60 \%$ of that of the $B_{0}=1$ tube. The plasma- $\beta$ is also smaller for higher values $B_{0}$ tubes, but the plasma- $\beta$ for the $B_{0}=9$ tube is $\sim 5 \%$ of the $B_{0}=1$ tube. This variation in the rate of decrease of the two terms with $B_{0}$ results in the left-hand term of (20), divided by the plasma- $\beta$, increasing as $B_{0}$ increases.
The convective term, given by the dashed line in Figs. 7a-e, appears to increase as the value of $B_{0}$ increases up to the $B_{0}=3$ case and then decreases as $B_{0}$ increases further. For the tubes with smaller values of $B_{0}$ this term dominates and, therefore, the tubes do not undergo the buoyancy instability. However, for the tubes with larger values of $B_{0}$ the magnetic term, given by the solid line in Figs. 7a-e, dominates and the tubes do suffer from the buoyancy instability and go on to emerge into the atmosphere.

The rightmost term of (20) is $\sim 0.025$ for all of the tubes and division by the plasma- $\beta$ at the top of the tube gives the range of values $1.25-0.0025$ for the tubes, with the larger valued $B_{0}$ tubes taking the larger values in the range. These values are given for completeness only since we find that this term does not alter the outcome of the criterion in anyway.

Finally, we find that the axes of tubes with larger values of $B_{0}$ reach greater heights within the photosphere. This is because the higher $B_{0}$ tubes have larger velocities and thus larger momentums when the plasma becomes over-dense, carrying the tube (including the axis) higher into the photosphere. The heights observed for the axes are not necessarily the heights at which they will come to rest since they are over-dense and not at equilibrium with the environment. However, within the limited time of the experiments it is not possible to determine the height at which they will finally settle.

\subsubsection{Expansion in the atmosphere}

As explained in Sect. 3.1.2, only three of the five cases under consideration see the tubes advance beyond the photosphere, namely $B_{0}=3.0,5.0$ and 9.0 (see Fig. 8). Within this section we will only be concerned with these tubes. Since the tubes experience different magnitudes of buoyancy forces, the crests of the tubes reach the photosphere, and start to advance into the upper atmosphere, at different times. By taking this time difference into consideration, we can make comparisons about the rate of rise of the tops of the tubes. We find that the tube with $B_{0}=9.0$ advances at the fastest rate and the tubes with $B_{0}=3.0$ and $B_{0}=5.0$ rise at the same rate as each other but at a slightly slower rate than $B_{0}=9.0$.

The rise speed of the tube in the atmosphere may be relatively independent of the strength of the tube but the amount of flux transported into the atmosphere is not. To quantify this, we take a horizontal plane at $z=10$ and measure the amount of unsigned vertical flux through this surface as a function of time. We find that the amount of flux in the atmosphere is directly proportional to $B_{0}$ and, by scaling time as $\bar{t}=t B_{0}$, we find a good agreement between the cases in the rate of the flux entering the upper atmosphere, Fig. 9.

\subsection{Group 2: varying $\alpha$ with fixed $B_{0}$}

\subsubsection{Rise in the solar interior}

The twist of the magnetic field of a tube results in a tension force acting on the tube. Modifying $\alpha$, the degree of twist, will alter the tension force and therefore change the buoyancy profile of the material in the tube. A comparison of the buoyancy profile for tubes with different values of $\alpha$ reveals that as the value of $\alpha$ is reduced the region around the axis becomes more buoyant, as shown in Fig. 10, but the plasma is less buoyant at outer radii due to the smaller magnetic field strength there.

We find that the axis of the tube with the smallest value of $\alpha$ reaches the top of the solar interior in the shortest amount of 
(a)

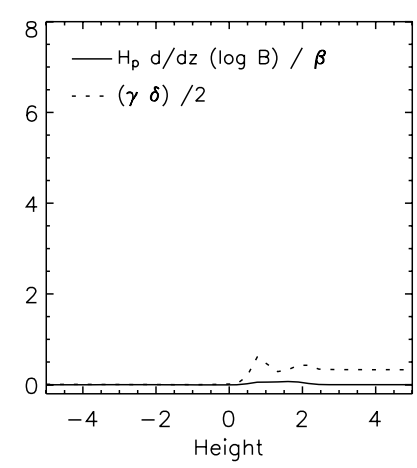

(d)

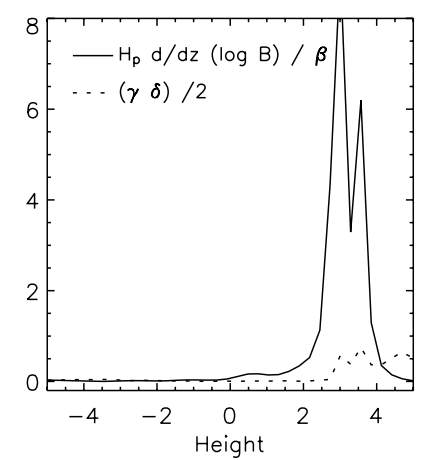

(b)

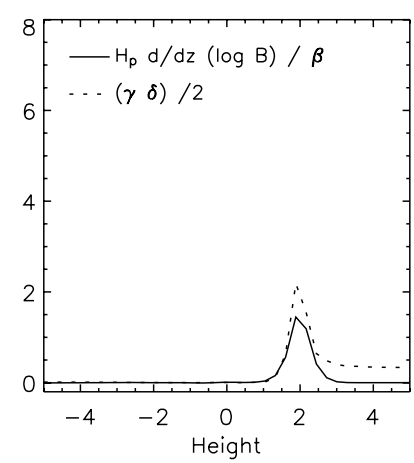

(e)

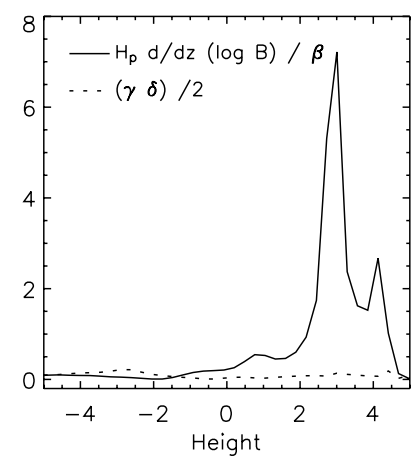

(c)

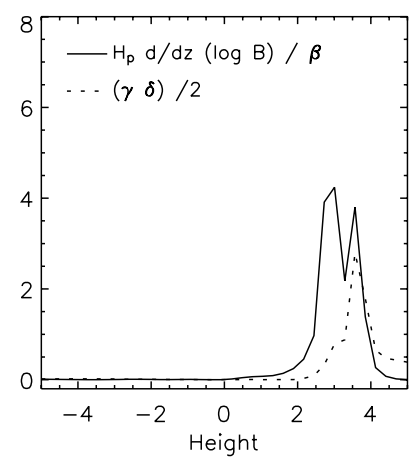

$(f)$

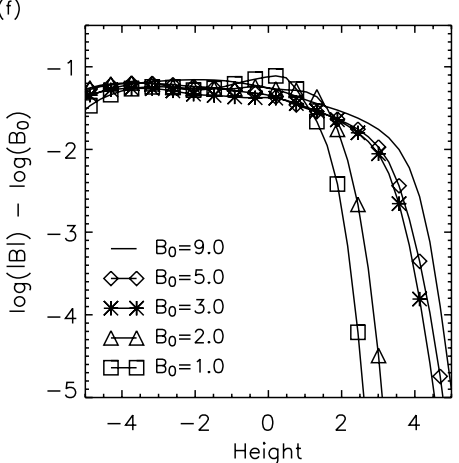

Fig. 7. a)-e): The left-hand side and first right-hand side terms of (20), divided by the plasma- $\beta$, for the various $B_{0}$ cases where a), b), c), d) and e) correspond to $B_{0}=1.0,2.0,3.0,5.0$ and 9.0 respectively, f) the natural logarithm of the magnitude of the magnetic field, where the magnitude of the field has been made independent of the initial field strength by subtracting the natual logarithm of $B_{0}$. The time at which each of the quantities in a)-f) are considered at varies with each tube and the times are $t=15,27,49,70$ and 152 for $B_{0}=9.0,5.0,3.0,2.0$ and 1.0, respectively. The quantities are measured along $(x=0, y=0)$ and are plotted against height.

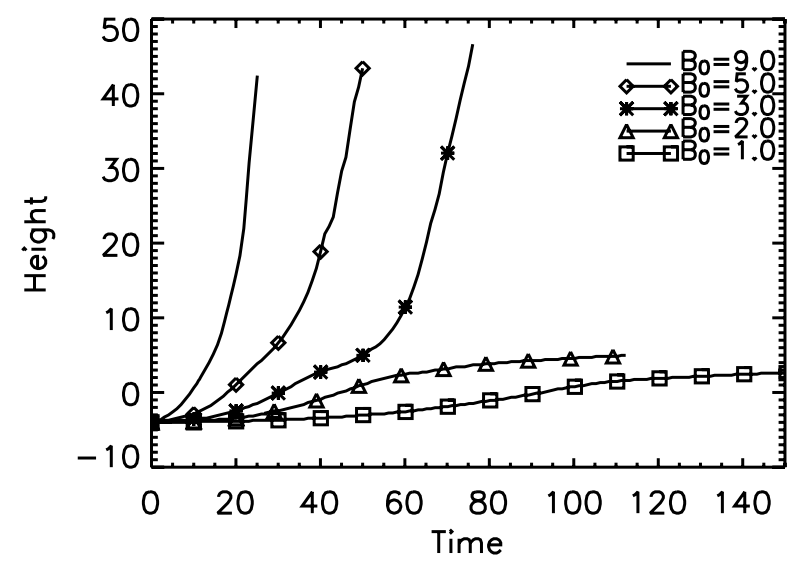

Fig. 8. The height-time relation of the top of the flux tube for the various cases of $B_{0}$.

time, Fig. 11a. This is because the tube has a larger buoyancy force at the axis and a lower tension force throughout. Given that the axis of the $\alpha=0.1$ tube is rising the quickest, it could be assumed that the front of this tube will also rise the quickest since the velocity of the material behind gives it momentum. However, Fig. 11b shows that there is little difference in the rate of rise of the fronts of the tubes during their rise though the solar interior for the various values of $\alpha$. These tubes exhibit no clearly quantifiable self-similarity during their rise through the solar interior, unlike the group 1 cases, due to the complicated manner in which the tension force acts upon the tube.

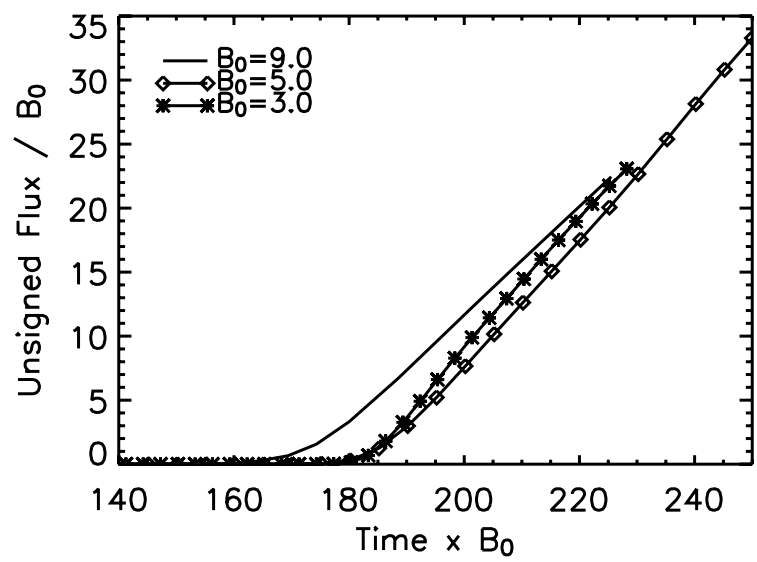

Fig. 9. The total vertical unsigned flux from the tube, measured at the transition plane $(z=10)$, as a function of rescaled time, $\bar{t}=t B_{0}$.

As discussed in Sect. 3.1.1 for the group 1 cases, as the buoyant section of the tube rises it compresses the slower moving material preceding it. This compression causes the azimuthal field, $B_{\theta}$, to increase in magnitude and the magnetic field lines of the tube to increase in pitch. Of the tubes under consideration in group 2, the faster rise of the axis of the $\alpha=0.1$ tube causes this tube to have the shortest distance between its axis and front. Therefore, the field directly above the axis of the tube is compressed to a greater degree than in the two tubes with larger $\alpha$ values. To evaluate this compression we consider the azimuthal field with height at $(x=0, y=0)$, which is solely the $B_{x}$ component of the field. To make comparisons between 


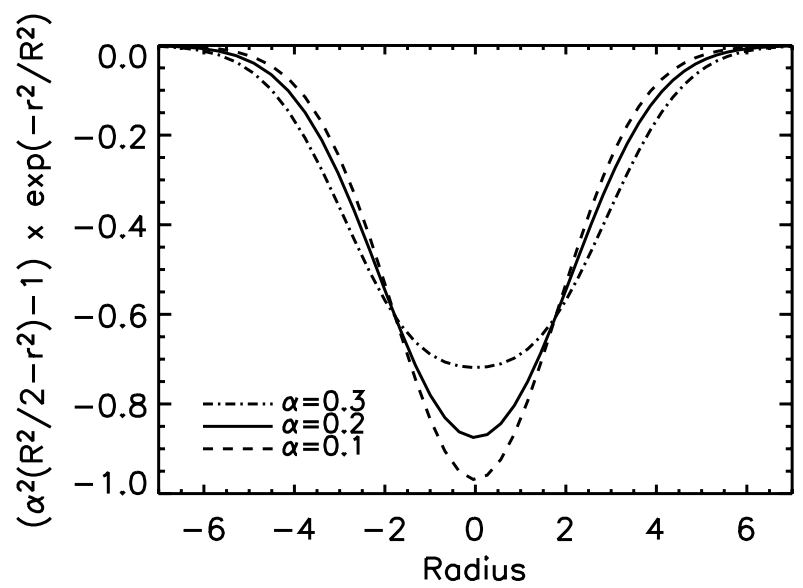

Fig. 10. From (14), a measure of the buoyancy of the tube for the various $\alpha$ cases.

the tubes we use a different time for each tube such that the top of the tube is just below the photosphere. Figure 12a illustrates an $83 \%$ increase in the maximum of the azimuthal field for the tube with $\alpha=0.1$ compared with only $48 \%$ and $14 \%$ increases for the $\alpha=0.2$ and 0.3 tubes respectively.

Whilst the azimuthal field is increasing in strength, the field in the axial direction is decreasing in magnitude due to the horizontal expansion experienced by the tube. The magnitude of the $B_{y}$ component of the field is independent of $\alpha$ at the beginning of the experiments, since all of the tubes in group 2 are chosen to have $B_{0}=2.9$. We find that the decrease in $B_{y}$, as a function of the height of the tube, occurs at the fastest rate for the $\alpha=0.1$ tube. As an illustration of this point, Fig. 13 shows the decrease in $B_{y}$, measured at the axis, compared with the height of the axis, $z_{a}$. The increased rate of decrease for the $\alpha=0.1$ tube occurs because this tube has the smallest tension force and therefore horizontal expansion is less constrained.

Although the tube with the smallest value of twist has the greatest degree of compression above the axis, the actual magnitude of the azimuthal field, when compared with the other tubes, is significantly smaller. The combination of the smaller azimuthal field and the fastest decreasing axial field results in the magnitude of the magnetic field for the $\alpha=0.1$ tube being the smallest and the gradient at the rising edge being the least steep, as shown by Fig. 12c. For the higher $\alpha$ tubes, the increase in the azimuthal field due to the compression allows this field component to become larger than the axial component. Thus it dominates the magnetic profile as the tube approaches the surface, as shown in Figs. 12a, b.

As already stated, the compression of the field causes the azimuthal component of the tube's field to increase and therefore the field lines turn further from the axial direction. For example, the smaller size of the azimuthal component of the field for the $\alpha=0.1$ tube means that the pitch of the field lines to the axial direction is smaller. As the crests of the tubes enter the photosphere, the pitch angle of their field lines is maintained and, therefore, there are noticeable difference between the horizontal orientation of each tube's field lines.

Many studies have shown that the field of an emerging flux tube must be twisted about its axis if it is to rise through the solar interior without suffering distortion. For the values of $\alpha$ being considered here, we find varying degrees of distortion in the shape of the tube. The cases of $\alpha=0.2$ and 0.3 maintain a radial decrease in the magnitude of the magnetic field for all $\theta$. This is (a)

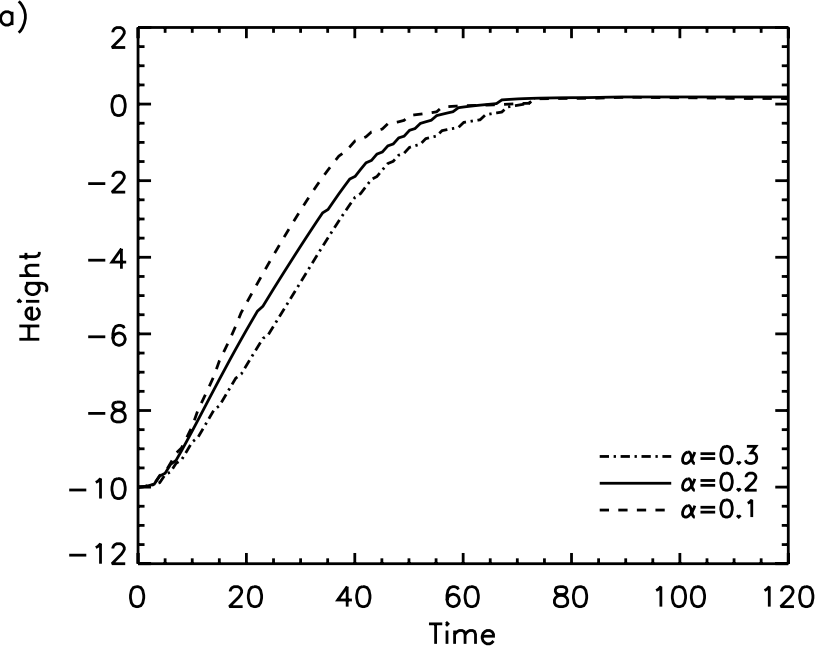

(b)

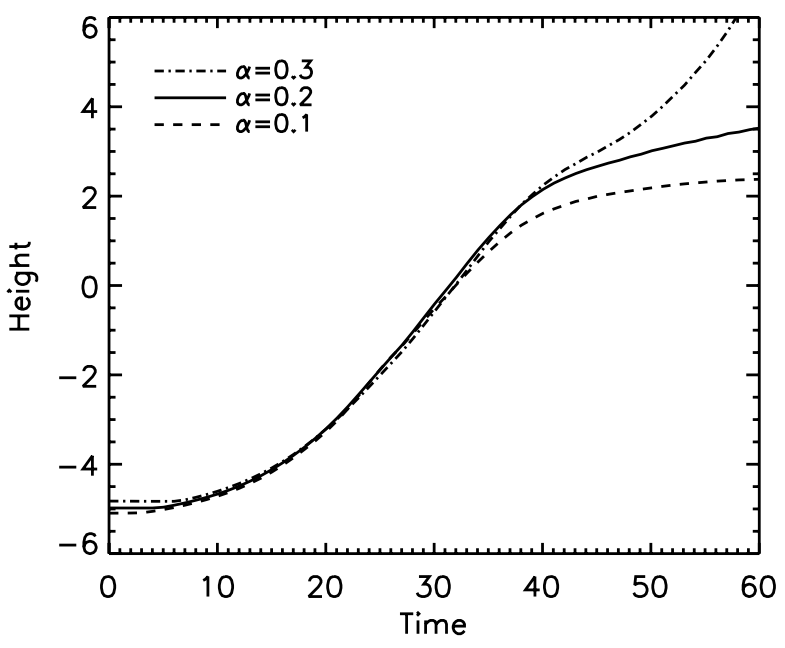

Fig. 11. Height-time relation of the a) axis and b) top of the tube for the various $\alpha$ cases.

not true for the $\alpha=0.1$ tube as shown by Fig. 14. The weaker tension force enables material to be dragged from the front of the tube towards the rear, as described by Moreno-Insertis \& Emonet (1996). Two side lobes begin to form and further distortion is prevented by the tube's arrival at the photosphere.

\subsubsection{Emergence into the photosphere}

Once the top of the tube reaches the photosphere, it is forced to advance at a slower pace due to the change in stratification. As discussed in Sect. 3.1.2, it is possible to lift over dense material into the atmosphere via the magnetic buoyancy instability. The values of $\alpha$ under consideration here give three different types of behaviour when the tops of the tubes reach the bottom of the photosphere. We find that the tube with $\alpha=0.1$ fails to emerge into the upper photosphere. However, the two tubes with larger values of $\alpha$ do emerge but with different configurations to each other.

For the $\alpha=0.3$ tube, it is the central portion of the tube in the axial direction which emerges first, giving rise to a dome shaped emerging front, Fig. 15a. The $\alpha=0.2$ tube exhibits a different emergence pattern. Portions of the tube either side of the central section lead the emergence into the upper photosphere, Fig. 15b. 
(a)

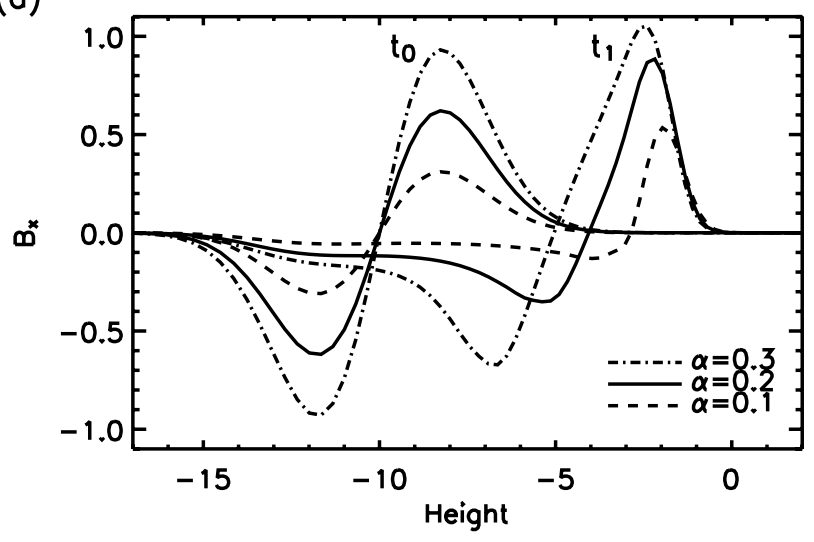

(b)

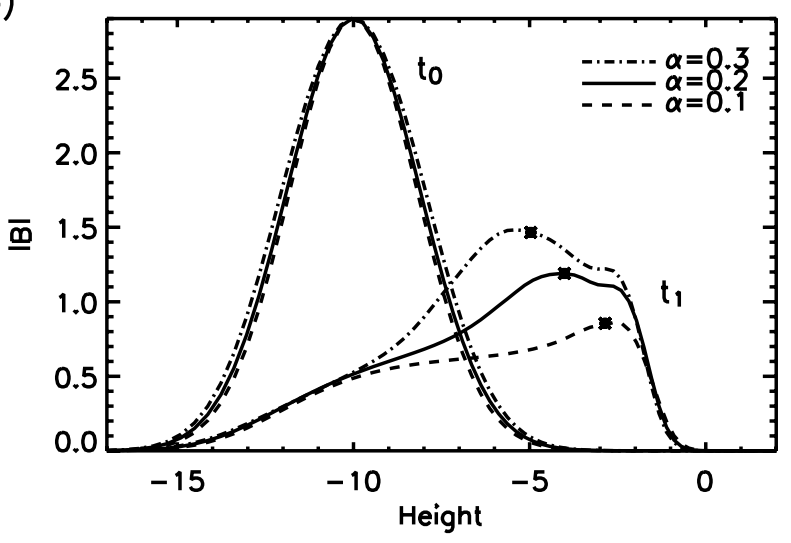

(c)

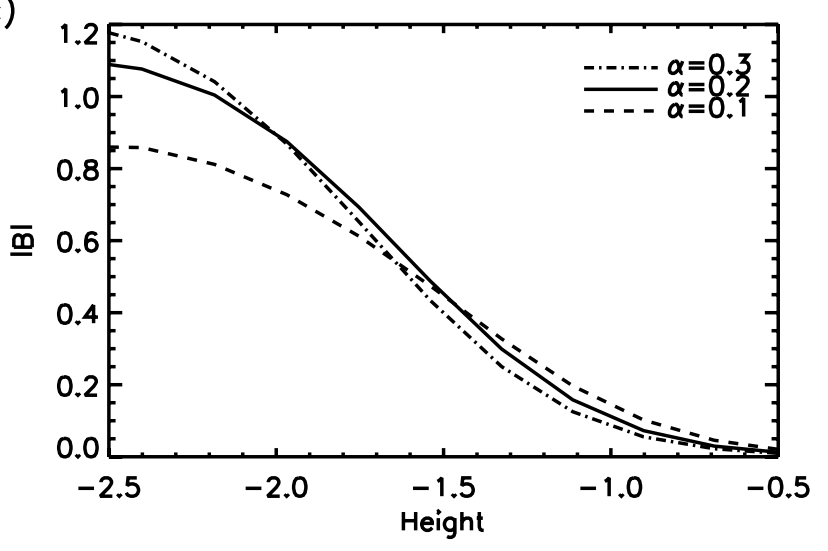

Fig. 12. a) Transverse component of the magnetic field, $B_{x}$, and b) magnitude of the magnetic field, $|B|$, measured with height at $(x=0, y=0)$ for the various $\alpha$ cases. The three lines labelled by $t_{0}$ in $\mathbf{a}$ ) and $\mathbf{b}$ ) are calculated at $t=0$. Those labelled by $t_{1}$ are at $t=29$ for $\alpha=0.1$ and $t=28$ for $\alpha=0.2$ and 0.3 . At these times the tops of the tubes are just approaching the photosphere. The stars on $\mathbf{b}$ ) indicate the height of the axes of the tubes at $t_{1}$. c) is a close-up of $\mathbf{b}$ ) for the region between the heights $z=-2.5$ and $z=0$.

For $\alpha=0.1$, we find that the tube does not emerge into the atmosphere and flattens out in a pancake shape at the solar interior photosphere interface, Fig. 15c.

The evolution of each individual tube is determined by the magnetic buoyancy instability criterion, (20). Figure 12c shows that the field at the front of the $\alpha=0.3$ tube has the steepest gradient when it reaches the photosphere. Additionally, the stronger field means the plasma- $\beta$ is smaller for the $\alpha=0.3$ tube both ini-

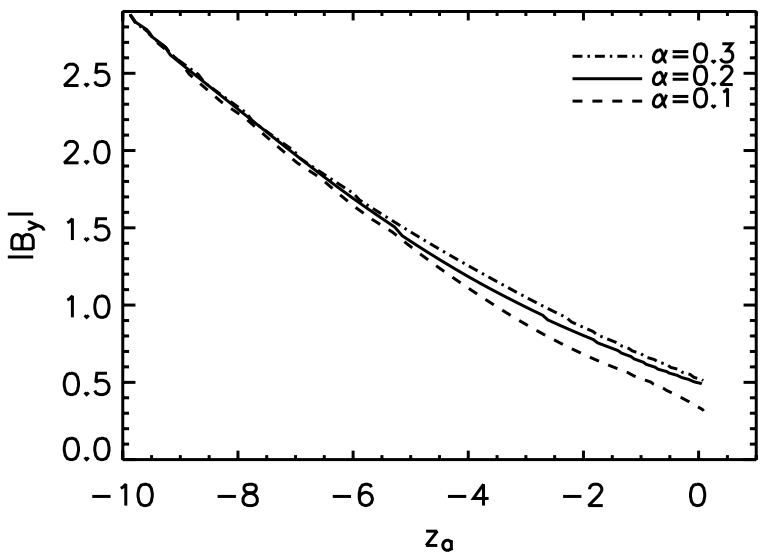

Fig. 13. The decrease in the $B_{y}$ component of the field at the axis plotted against the height of the axis, $z_{a}$, for the various $\alpha$ cases.

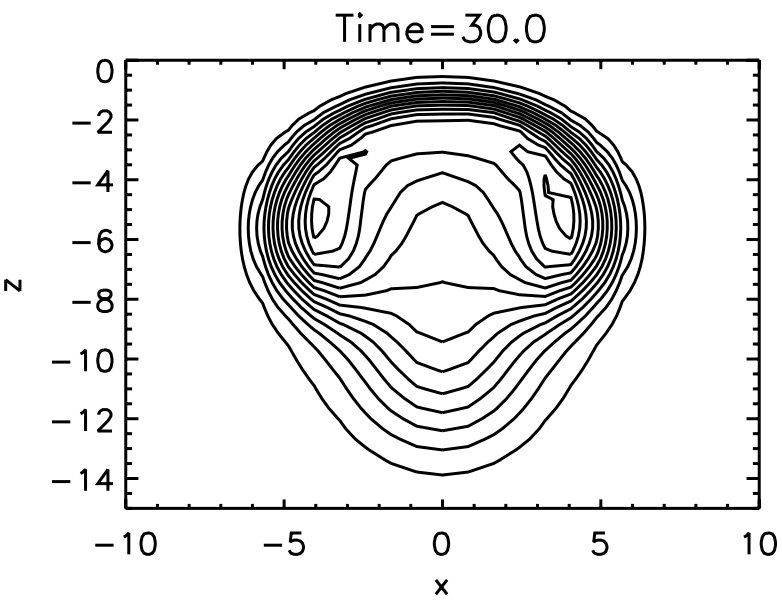

Fig. 14. Contours of the magnitude of the field strength of the $\alpha=0.1$ tube, $|B|$, viewed in the transverse cross-sectional plane $(y=0)$ at time $t=30$.

tially and when it arrives at the photosphere. Thus, (20) is easily satisfied at the crest of the tube and the emergence proceeds.

The strength of the field is greatly reduced for the $\alpha=0.1$ tube and it has the least steep field gradient at its front. The front of this tube also does not rise as high into the photosphere as the other two cases and, therefore, the external gas pressure remains slightly higher and the plasma- $\beta$ cannot be reduced by this secondary route. The combination of these two effects results in this tube not being able to emerge into the atmosphere.

For $\alpha=0.2$, the tube front reaches the same height as the $\alpha=0.3$ case and, therefore, experiences the same external gas pressure. However, the plasma- $\beta$ does not fall as low as for the $\alpha=0.3$ tube because the field strength of the tube is smaller. Figure 16a shows that the field compression and convective terms of the buoyancy criterion, (20), are practically equal at the central location. However, with the inclusion of a value of 0.05 for the rightmost term of (20) divided by the plasma-beta at the top of the tube, the buoyancy criterion is not satisfied. To understand why emergence is possible at the two side locations for this tube, we measure the quantities comprising (20) at these locations, namely $(x=0, y=-19)$ and $(x=0, y=20)$. 


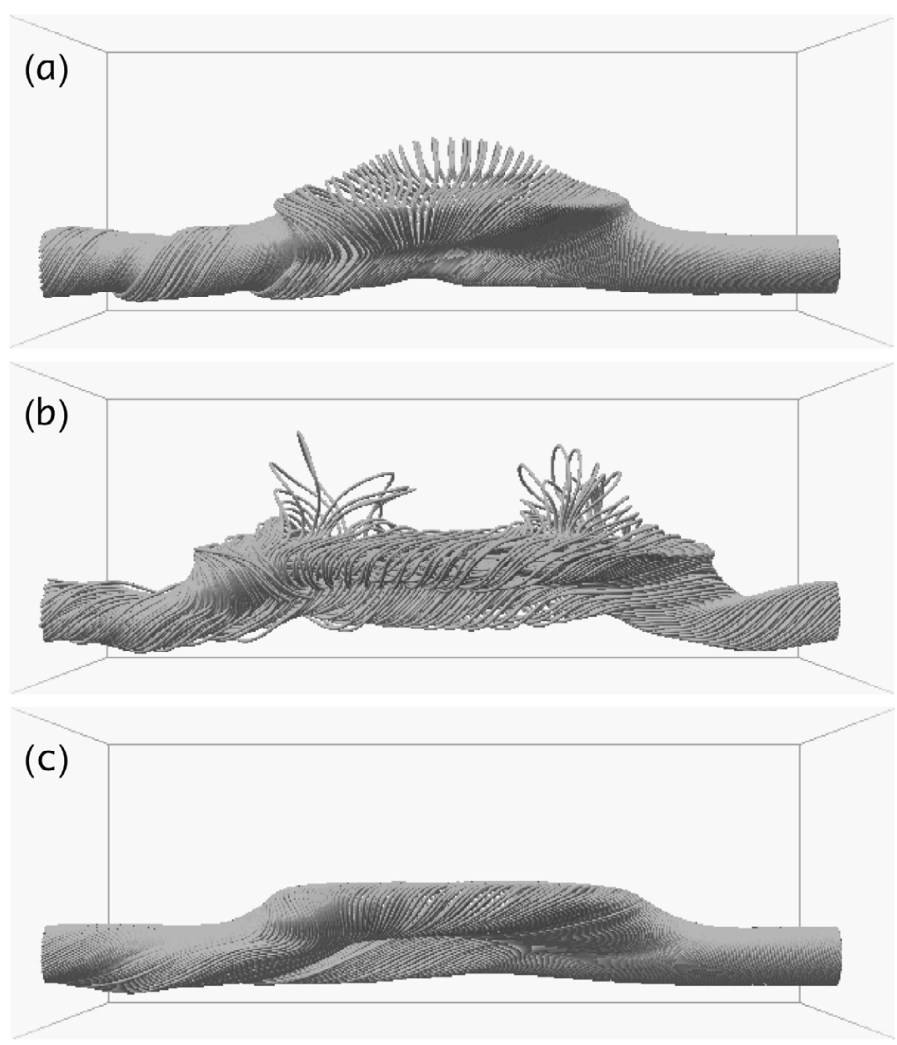

Fig. 15. Axial view of the outer field lines of the flux tube for a) $\alpha=0.3$ at $t=65$, b) $\alpha=0.2$ at $t=104$ and c) $\alpha=0.1$ at $t=70$.

At the height where we find the instability occurs, namely $z=2.7$, we find that the magnitude of the magnetic field takes the same value at all three locations, as shown by Fig. 16d. The gas pressure at this height is also very similar for all three locations and, therefore, there is little variation in the value of the plasma- $\beta$ at $z=2.7$.

Additionally, we find no variation in the gradient of the magnitude of the field at $z=2.7$ at the three locations although there is variation both at lower and higher heights. The gradient in the gas pressure is marginally steeper for these two side locations around the height where the instability takes hold, thus the pressure scaleheight, $H_{\mathrm{p}}$, is smaller than in the central location. The smaller $H_{\mathrm{p}}$ results in the left-hand term of (20) being smaller at $z=2.7$ for the two side locations, as confirmed by Fig. 16a-c.

Panels (a)-(c) of Fig. 16 identify that the convective term, right-hand term of (20), is smaller at the two side locations. This term is controlled by the gradient in the logarithmic temperature and, since temperature is defined by gas pressure and density via the ideal gas law, we can determine why the convective term varies by considering these alternative quantities. Above we have stated that the gas pressure takes the same values for the three locations at a height of $z=2.7$ and that there is little variation in the gradient of the gas pressure, thus we turn our attention to the density.

For all of the tubes in groups 1 and 2, we find downflows along the outer most field lines when the tube top flattens out at the photosphere, as indicated by the black arrows in Fig. 17a for the $\alpha=0.2$ case. For the tubes whose central portion emerges into the atmosphere, these downflows are maintained due to the draining of plasma as the tube expands into the atmosphere. However, for the tubes that do not undergo the buoyancy instability at $y=0$, we find the draining at this central location ceases as the field here continues to flatten. As the rest of the tube continues to rise up to the photosphere, the location of the draining moves outwards in the positive and negative $y$ directions and reduces in intensity. The black arrows in Fig. 17b identify the shift in draining locations over a period of 8 time units for the $\alpha=0.2$ case. The twisted nature of the tube's field means the plasma drained around $y=0$ accumulates in the troughs of the field lines in the centrally risen portion of the tube. However, the plasma draining around $y= \pm 20$ sinks down to the footpoints of the $\Omega$-loop in the solar interior where we find an accumulation of dense plasma.

At the times considered in Figs. 16a-c for the $\alpha=0.2$ tube, we find that there is a greater difference between the density at the top of the tube and the environment in the central location than at the two side regions. The enhanced reduction in density in the central portion occurs since the draining is occuring at a faster rate here. At a height of $z=2.7$, the gradient in the density at the two side locations is larger thus the convective term is smaller here. Hence, emergence ensues at the two side locations as a result of the marginal variations in the gas pressure gradient and the larger variations in the density gradient.

Independent of whether or not the tubes of the group 2 cases emerge, we find the evolution of the tubes has several common properties. Firstly, the slow down in the rise speed of the crest of the tube, as it approaches the photosphere, forces the vertically rising plasma in the remainder of the tube to spread out horizontally at the photosphere. Figure 18 shows that the growth of the photospheric area containing flux from the tube occurs at a rate that is mostly independent of $\alpha$. This comparable rate of coverage occurs because the tops of the tubes rise through the solar interior with the same speed and all of the tubes experience a similar braking effect upon reaching the photosphere, as discussed in Sect. 3.2.1. Secondly, regardless of the amount of twist in the tube, the axes of the tubes tend to the same height, namely $z=0$.

\subsubsection{Expansion in the atmosphere}

As stated in Sect. 3.2.2, only two of the three tubes in the group 2 cases emerge above the photosphere and these have values of $\alpha=0.2$ and $\alpha=0.3$. In this section we will be considering just these two cases.

We find that although the tops of the tubes reach the photosphere at the same time, the tube with $\alpha=0.2$ spends longer in the photosphere while waiting for the buoyancy instability to occur, see Fig. 19. Results from a simulation by Archontis et al. (2005) fit well with the height-time relation shown here. Their study considered an emerging tube with $\alpha=0.4$ but all other tube parameters remain the same as those used in this series of simulations. Independent of the value of $\alpha$ the tube reaches the base of the photosphere at a time $t \sim 30$ and, as expected, the $\alpha=0.4$ tube reaches the transition region at an earlier time, $t \sim 60$. The top of this tube spends less time in the photosphere since it experiences a greater degree of compression of the magnetic field at the tube front and, therefore, the buoyancy instability occurs at an earlier time.

We now return to the $\alpha=0.2$ and $\alpha=0.3$ tubes. Taking into account the different lengths of time the tops of the two tubes spend waiting for the buoyancy instability to develop, we find that once the highest emerging point of the tubes reaches the transition region the further rise of this point to the lower corona occurs at the same rate. To quantify the emergence of flux into the atmosphere we consider a horizontal plane at the start of the transition region, $z=10$. A comparison of the area of this plane containing flux from the tube, Fig. 20a, identifies that when the tops of the tubes are at the same height within the 
(a)

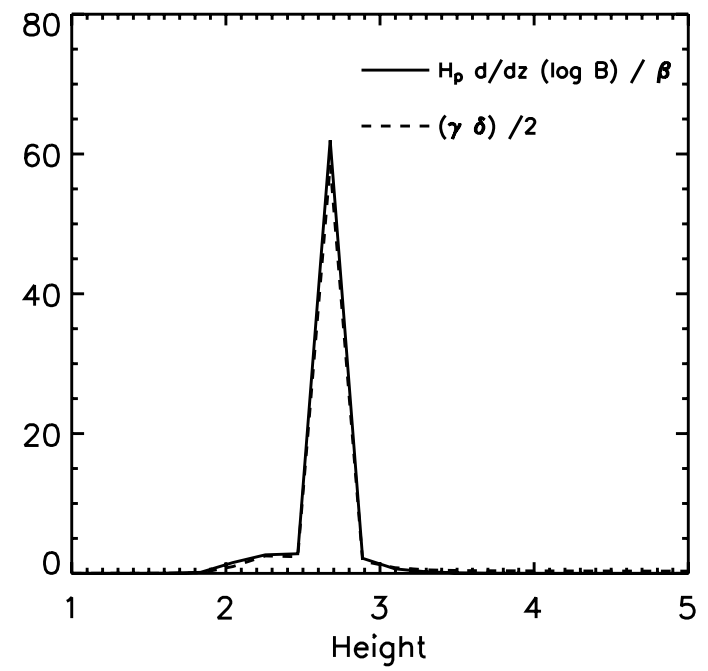

(c)

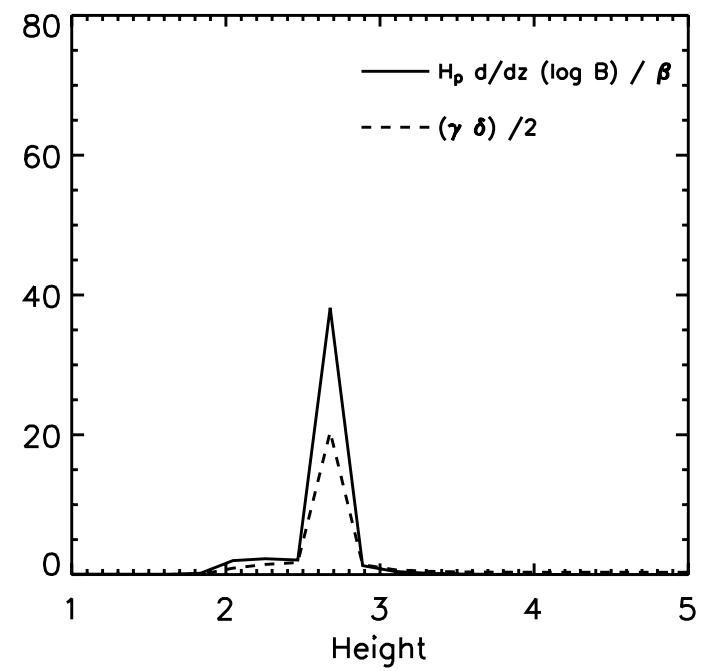

(b)

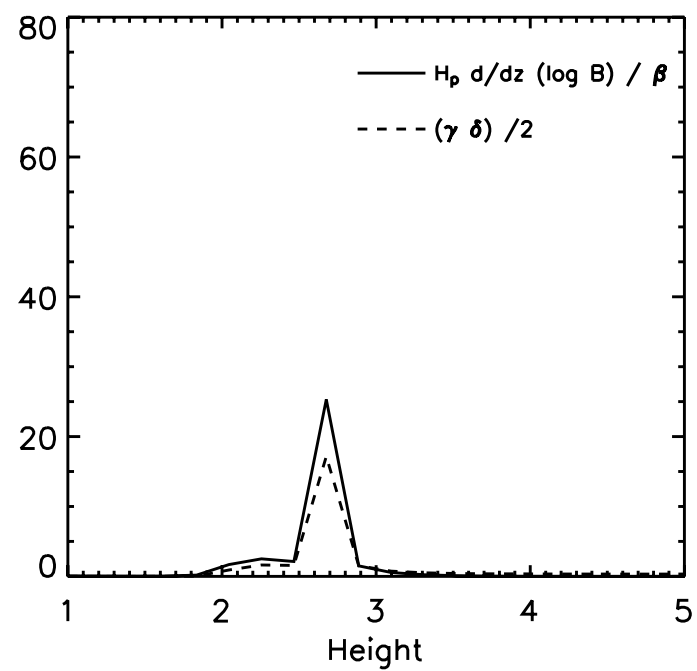

(d)

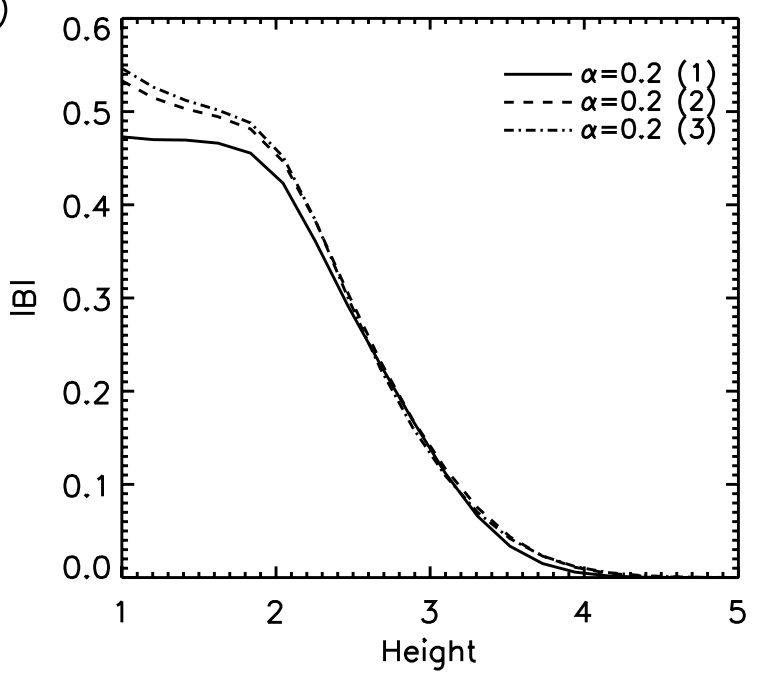

Fig. 16. a)-c): The left-hand side and first right-hand side terms of (20), divided by the plasma- $\beta$, for the $\alpha=0.2$ case measured at three separate locations. a), b) and c) correspond to measurements in height at $(x=0, y=0)$ at time $t=60,(x=0, y=-19)$ at $t=71$, and $(x=0, y=20)$ at $t=72$ respectively. d) The magnitude of the magnetic field in height at the same three locations where (1), (2) and (3) correspond to $(x=0, y=0)$, $(x=0, y=-19)$ and $(x=0, y=20)$ and the specific times are the same as for $\mathbf{a})-\mathbf{c})$.

transition region the same sized area of the plane is occupied. However, as the tubes enter the mid-corona, a height of $z \sim 27$, we see a difference in both the rise rate of the top of the tube and the size of the transition region plane occupied by flux.

For the $\alpha=0.2$ tube there comes a time when the boundaries of the two emerging domes will come into contact with each other and this occurs when the domes have reached a height of $z \sim 27$ at 104 time units. At this time we see a plateau in the rise of the tube, which occurs while the domes expand horizontally to fill any gaps left between themselves at the touching boundaries. After this, the rise continues at a similar rate to that of the tube rising through the transition region and the rise of the $\alpha=0.3$ tube. However, the area of the transition region plane containing tube flux is now significantly higher for the $\alpha=0.2$ tube and, although the areas occupied by flux increase with height at the same rate for both the tubes as they continue to rise through the mid-corona, once the tubes reach a height of $z \sim 35$ the area occupied by the $\alpha=0.3$ tube is just five-eighths that of the $\alpha=0.2$ tube. Although the highest point of the tubes are rising at the same rate, the difference in the occupied area of the plane occurs because the emergence of the $\alpha=0.2$ tube is being driven from two locations in comparison to just one for the higher $\alpha$ tube. We note that the difference in the area is almost $1 / 2$ and, therefore, conclude that the rates of expansion of the two emerging fronts of the $\alpha=0.2$ tube are practically the same as that of the single front of the $\alpha=0.3$ tube.

Figure 20b shows that the total unsigned flux emerging into the atmosphere, measured at the transition region plane, occurs at a faster rate for the tube with $\alpha=0.3$ even though the tops of the emerging domes, one in the case of $\alpha=0.3$ and two for $\alpha=0.2$, are rising into the atmosphere at the same rate. When the field was at the photosphere, the magnetic strength was smaller for the $\alpha=0.2$ tube, thus the field being transported into the atmosphere is also weaker and this manifests itself as a slower rate of increase in the total unsigned flux.

\section{Conclusions}

In this paper, we presented results from 3D MHD simulations of buoyant flux tubes rising through the solar interior and emerging into the atmosphere. We have varied the values of two of 
(a)

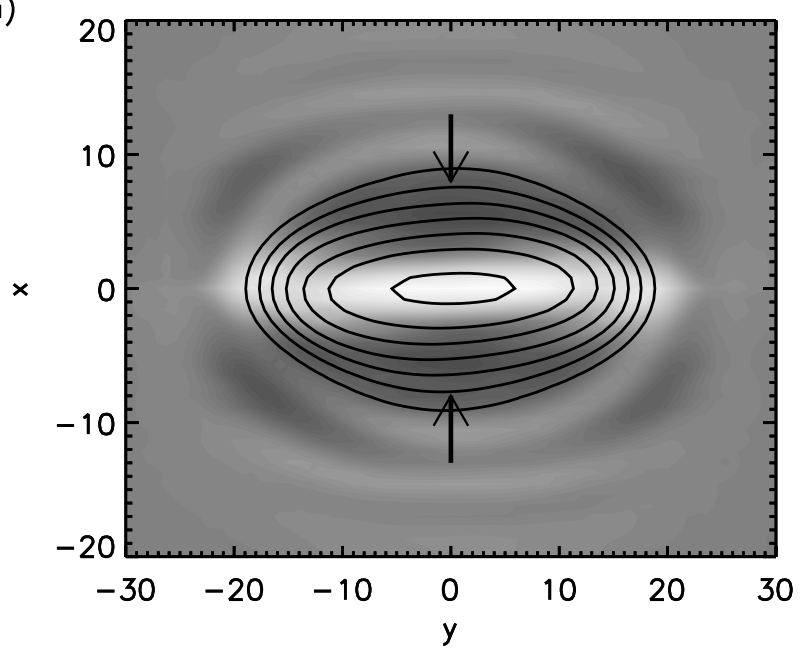

(b)

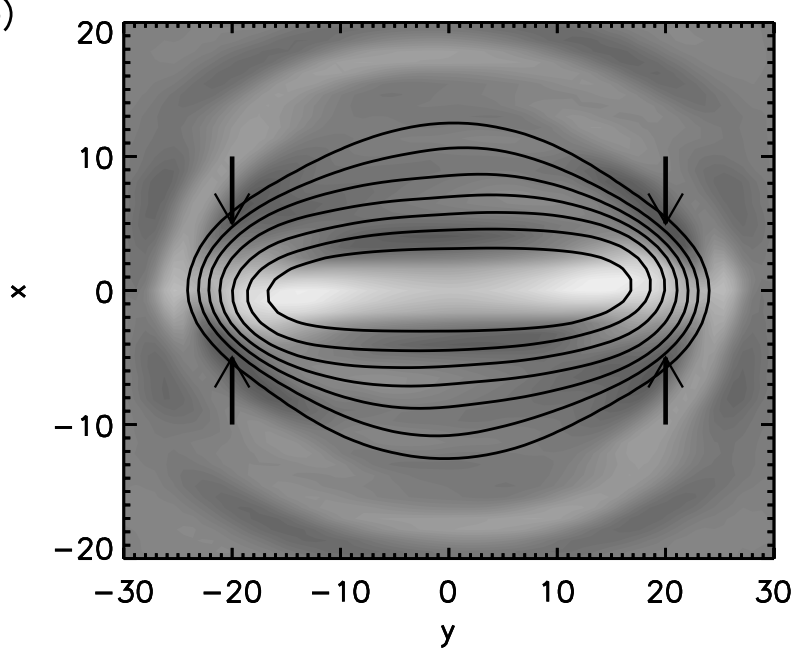

Fig. 17. Grey-scale map of vertical velocity measured in the horizontal plane at a height of $z=1.8$ for the $\alpha=0.2$ tube. a) and b) correspond to times $t=50$ and $t=58$, respectively. The colouring in both a) and $\mathbf{b}$ ) is scaled such that white indicates a maximum upflow of $v_{z}=$ $0.046 H_{\mathrm{ph}} t_{\mathrm{ph}}^{-1}$ and dark grey indicates a maximum downflow of $v_{z}=$ $-0.047 H_{\mathrm{ph}} t_{\mathrm{ph}}^{-1}$. The black elliptical contours are of $|\boldsymbol{B}|$ and give the location of the tube, which dissects the plane. The black arrows indicate specific regions of downflow within the tube itself.

the parameters defining the magnetic structure of the flux tube, namely the magnetic field strength, $B_{0}$, and the twist of the tube's field, $\alpha$. Our aim was to understand the individual effect of each on the emergence process.

When varying the magnetic field strength of the tube, we have found self-similarity in the tube's evolution. When the difference in the strengths of the tubes is taken into consideration, we find that the rise time through the solar interior, the increase in the area of the photospheric plane containing flux from the tube, and the increase in unsigned flux into the atmosphere each occur at the same rate for all the tubes. The velocity and magnetic strength of the axes of the tubes also show self-similar behaviour with dependence upon $B_{0}$ being easily removed with appropriate scaling. Over the initial rise period the decrease in the strength of the axes of the tubes is described reasonably well by the thin flux tube approximation.

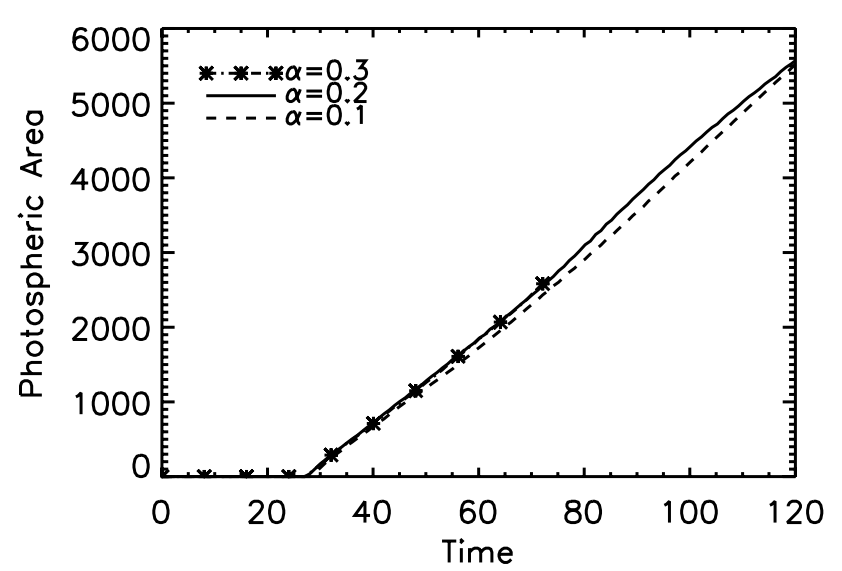

Fig. 18. Measurements of the area of the photospheric plane $(z=0)$ containing flux from the emerging tube for the various $\alpha$ cases as a function of time.

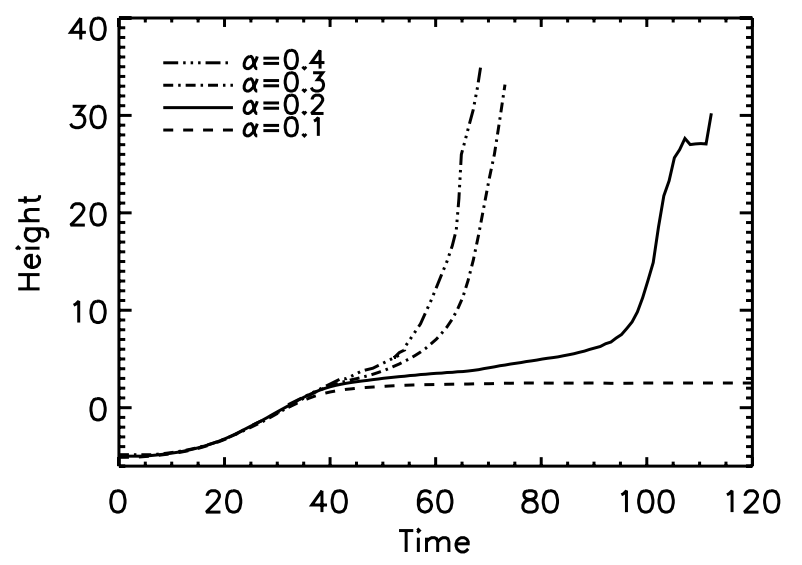

Fig. 19. Height-time function of the top of the tube for the various $\alpha$ cases. The triple-dot dashed line giving information for the $\alpha=0.4$ case is a result obtained in a simulation by Archontis et al. (2005) where a tube emerges into an unmagnetised corona.

We find that if the magnetic field strength of the tube is initially too small, the magnetic buoyancy instability does not occur when the tube reaches the top of the solar interior and, therefore, it is not possible for the tube to expand into the atmosphere. The magnetic buoyancy instability criterion (20) is valid when considering a static equilibrium situation and is not strictly applicable when the tube is rising, as in our cases. However, in this study the stronger tubes have only small vertical velocities associated with them $(\boldsymbol{v} \ll$ Alfvén speed) and so the criterion holds approximately. We have shown that it correctly predicts which tubes will emerge and which will not by checking the relative sizes of the magnetic and convective terms, thereby justifing its use.

We find that the case with $B_{0}=9.0$ exhibits different behaviour to the other tubes and fits less well with the self-similar advancement of the tubes. When this tube is initialised in the upper layers of the solar interior the plasma- $\beta$ of the material in the tube is considerable lower than the other cases due to the large magnitude of the tube's magnetic field. There is over two orders of magnitude difference between the plasma- $\beta$ of the $B_{0}=9.0$ 


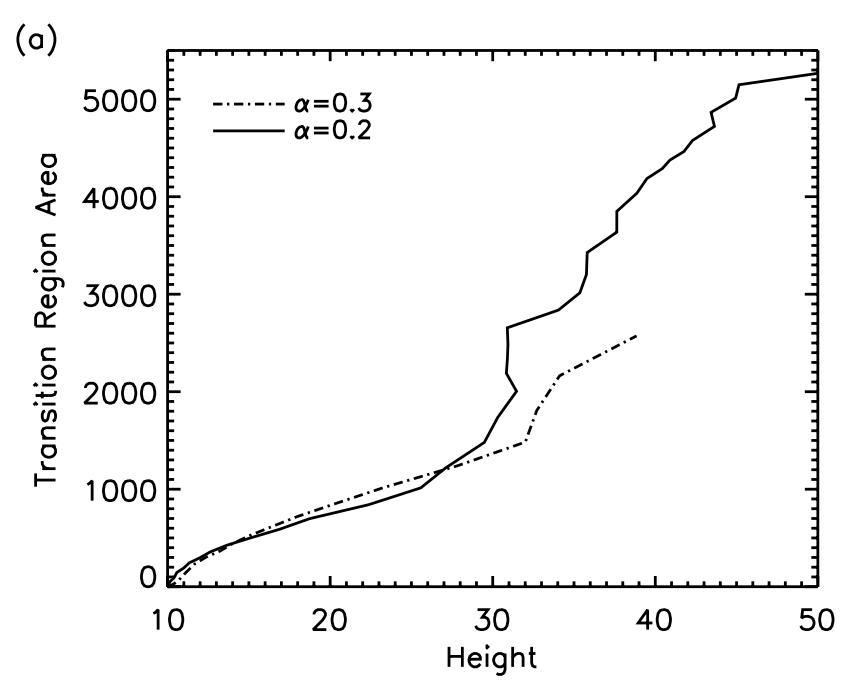

(b)

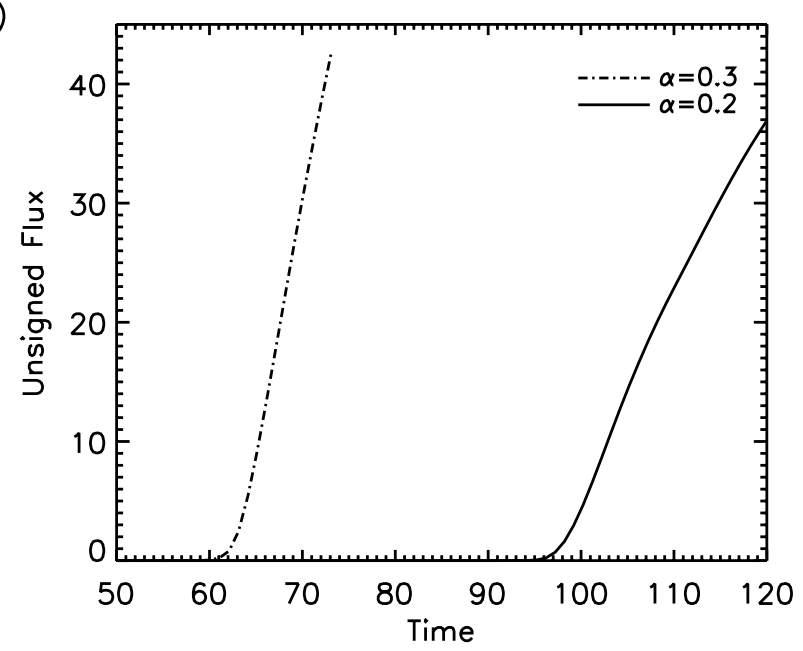

Fig. 20. Measured at the transition region plane $(z=10)$, a) the area containing flux from the tube and b) the total unsigned vertical flux from the tube as functions of time.

and $B_{0}=1.0$ tubes. We believe that the lower plasma- $\beta$ in the $B_{0}=9.0$ case, a value of only 0.9 at the axis, causes this tube's evolution to be dominated by the magnetic forces whilst the gas pressure and density play a more active role in determining the progression of the other tubes.

The variation in the twist of the flux tube does not result in such clearly quantifiable self-similar behaviour during the rise through the solar interior. The lower value of twist for the $\alpha=0.1$ tube allows for distortion in the shape of the tube during its rise and, thus, when it approaches the photosphere the field is too weak to enable the buoyancy instability to occur. This tube can only flatten out in the lower photosphere and does not advance into the upper atmosphere. The usual pattern of emergence, led from the crest of the tube, is seen for the higher twist tube. For the first time, we see emergence of a buoyant flux tube occurring away from the crest of the tube for a value of twist that is neither too weak nor too strong. The emergence of flux from the $\alpha=0.2$ tube at two side locations occurs as a result of draining of plasma from the upper part of the tube. This may suggest that flux tubes with weaker magnetic magnitude, but which are more highly twisted, may be able to emerge through a similar process of draining.

Although we see multiple differences when the twist of the flux tube is varied, we also see several similarities between the experiments. The rate of expansion of the tube at the photosphere and the rate of expansion of any emerging tube sections each occur at similar rates.

The effects of varying the magnetic field strength and twist of a flux tube, which we have discussed, raise several further questions beyond the scope of this paper. In these experiments the atmosphere is unmagnetised but this is certainly not the case with the Sun's corona. How would the faster rise of the stronger flux tubes affect the reconnection between two correctly aligned flux systems? Would reconnection between a magnetised atmosphere and a tube be complicated by flux emerging at two locations from the tube? Flows in the photospheric domain have been shown to propagate through to the corona so would the variation in rise speed of the emerging subphotospheric flux cause variations in the corona? Further modelling of flux emergence from the solar interior into the atmosphere will be key to obtaining a better understanding of the dynamic, complicated, and visible processes occurring in the Sun's atmosphere.

\section{References}

Abbett, W. P. \& Fisher, G. H. 2003, ApJ, 582, 475

Abbett, W. P., Fisher, G. H., \& Fan, Y. 2000, ApJ, 540, 548

Acheson, D. J. 1979, Sol. Phys., 62, 23

Archontis, V., Moreno-Insertis, F., Galsgaard, K., Hood, A., \& O’Shea, E. 2004, A\&A, 426, 1047

Archontis, V., Moreno-Insertis, F., Galsgaard, K., \& Hood, A. W. 2005, ApJ, 635, 1299

Cheung, M. C. M., Moreno-Insertis, F., \& Schuessler, M. 2006, A\&A, 451, 303

Dorch, S. B. F. \& Nordlund, A. 1998, A\&A, 338, 329

Dorch, S. B. F. 2003, in The Future of Cool-Star Astrophysics: 12th Cambridge

Workshop on Cool Stars, Stellar Systems, and the Sun (2001 July 30-August

3), ed. A. Brown, G.M. Harper, and T.R. Ayres, (University of Colorado), ed.

A. Brown, G. M. Harper, \& T. R. Ayres, 186

Dorch, S. B. F., Archontis, V., \& Nordlund, A.. 1999, A\&A, 352, L79

Dorch, S. B. F., Gudiksen, B. V., Abbett, W. P., \& Nordlund, Å. 2001, A\&A, 380,734

Emonet, T. \& Moreno-Insertis, F. 1998, ApJ, 492, 804

Fan, Y. 2001, ApJ, 554, L111

Fan, Y. 2004, Living Rev. Sol. Phys., 1, 1

Fan, Y., Zweibel, E. G., Linton, M. G., \& Fisher, G. H. 1998, ApJ, 505, L59

Fan, Y., Abbett, W. P., \& Fisher, G. H. 2003, ApJ, 582, 1206

Galsgaard, K., Moreno-Insertis, F., Archontis, V., \& Hood, A. 2005, ApJ, 618, L153

Kusano, K., Moriyama, K., \& Miyoshi, T. 1998, Phys. Plasmas, 5, 2582

Leake, J. E. \& Arber, T. D. 2006, A\&A, 450, 805

Leka, K. D., Canfield, R. C., McClymont, A. N., \& van Driel-Gesztelyi, L. 1996, ApJ, 462, 547

Magara, T. 2001, ApJ, 549, 608

Magara, T. \& Longcope, D. W. 2001, ApJ, 559, L55

Manchester, W., Gombosi, T., DeZeeuw, D., \& Fan, Y. 2004, ApJ, 610, 588

Matsumoto, R. \& Shibata, K. 1992, PASJ, 44, 167

Matsumoto, R., Tajima, T., Shibata, K., \& Kaisig, M. 1993, ApJ, 414, 357

Miyagoshi, T., Isobe, H., Shibata, K., \& Yokoyama, T. 2006, in preparation

Miyagoshi, T. \& Yokoyama, T. 2004, ApJ, 614, 1042

Moreno-Insertis, F. 2005, in ASP Conf. Ser.: Solar MHD: Theory and Observations - a High Resolution Perspective, ed. J. Leibacher, H. Uitenbroek, \& R. Stein

Moreno-Insertis, F. \& Emonet, T. 1996, ApJ, 472, L53

Newcomb, W. A. 1961, Phys. Fluids, 4, 391

Nordlund, A. \& Galsgaard, K. 1997, A 3D MHD Code for Parallel Computers,

Tech. rep., Astronomical Observatory, Copenhagen University

Ryutova, M. \& Shine, R. 2006, J. Geophys. Res. (Space Phys.), 111, 3101

Thomas, J. H. \& Nye, A. H. 1975, Phys. Fluids, 18, 490

Yu, C. P. 1965, Phys. Fluids, 8, 650 